\title{
Functionalized Fluorescent Nano-dots for Discrimination of Nitroaromatic Compounds
}

\author{
Pradipta Behera, Ashutosh Mohanty and Mrinmoy De
}

${ }^{a}$ Department of Organic Chemistry, ${ }^{b}$ Solid state and Structural Chemistry Unit, Indian Institute of Science, Bengaluru-560012, India

Email:md@iisc.ac.in 


\section{Experimental Section}

Reagents: $\mathrm{MoS}_{2}$ and $\mathrm{WS}_{2}$ bulk powders were purchased from Sigma-Aldrich Corporation. All the nitro aromatic compounds, metal salts, $\mathrm{HAuCl} 4$ and Glutathione were purchased from SRL, SDF, Thomas Backer and Avra Chemicals India.

Synthesis of gold nanocluster (AuNC): AuNC were synthesized by taking $\mathrm{HAuCl}_{4}$ as gold precursor and glutathione (GSH) as capping cum reducing agent. Freshly prepared $0.5 \mathrm{~mL}$ of $\mathrm{HAuCl}_{4}(20 \mathrm{mM})$ and $0.15 \mathrm{~mL}$ of $\mathrm{GSH}(100 \mathrm{mM})$ were added to $4.35 \mathrm{~mL}$ of ultrapure water at room temperature. After that, whole solution was stirred at $500 \mathrm{rpm}$ at $70^{\circ} \mathrm{c}$ for 24 hours. The resultant pale-yellow color solution was stored at $4^{\circ} \mathrm{c}$ for further use.

Synthesis of TMDQDs: Both MQD and WQD were prepared through top-down approach. About $300 \mathrm{mg}$ of bulk TMD powders in $30 \mathrm{~mL}$ of DMF were sonicated by using tip sonicator for 30 minutes followed by heating at $140^{\circ} \mathrm{c}$ for 6 hours. After that, the above solution was centrifuged at $10,000 \mathrm{rpm}$ for 30 minutes to remove bigger size particle and sheets. Pale-yellow color supernatant was collected and concentrated in vaccum to remove DMF. Resultant solid was re dispersed in water and stored at $4^{\circ} \mathrm{c}$ for further use.

Functionalization of all quantum dots: About $10 \mathrm{mg}$ of as prepared thiol ligand was mixed 8 $\mathrm{mL}$ of water and $2 \mathrm{~mL}$ of quantum dots solution followed by stirring for 48 hours at room temperature. After that, whole solution was washed with ethyl acetate to remove free thiol ligands. Thiol-functionalized quantum dots were stored at $4^{\circ} \mathrm{c}$ for future application.

Zeta Potential Measurements: Zeta potential measurement of all native and functionalized quantum dots were done by using MALVERN ZETASIZER Nano ZS. Quantum dot solutions were diluted five times in ultrapure water and $800 \mu \mathrm{L}$ of it was taken in a zeta cell for zeta potential measurement.

UV-Vis Spectroscopy: Diluted solution of all quantum dots and NACs in ultrapure water were used for absorption spectroscopy. All the spectral recordings were done by using UV-Vis-NIR Spectrophotometer (Japan Analytical Instrument) and quartz cuvette with $10 \mathrm{~mm}$ path length and $1 \mathrm{~mL}$ volume capacity.

Fluorescence measurement: All the fluorescence experiments were done in 96 black flat bottomed microwell plate by using VARIOSKAN multimode plate reader instrument. $190 \mu \mathrm{L}$ of quantum dots solution was taken in each well and their fluorescence intensity was recorded and termed as $\mathrm{I}_{0}$. To the quantum dots, stock solution of analytes (NACs), quenching agent (turn-Off) and masking agent (turn-On) were added sequentially and their fluorescence response were termed as $I_{1}, I_{2}$ and $I_{3}$ respectively. For each quantum dot, three response i.e. $\left(I_{0}-I_{1}\right),\left(I_{1}-I_{2}\right)$ and $\left(I_{3}-I_{2}\right)$ were collected and used as fluorescence response pattern and dataset for LDA analysis. Concentration of stock solution was n-times higher than that of working concentration. $\mathrm{n}=20$ for analytes, 21 for QA, 22 for MA. 
TEM characterization: All the TEM and HRTEM images were recorded by using JEOL-JEM 2100F microscope at $200 \mathrm{KeV}$ electron energy. TEM sample were prepared by drop casting 10 $\mu \mathrm{L}$ of quantum dot solutions on top of CF300-Cu carbon film on 300 mesh copper grids and allowed for drying overnight.

\section{Fluorescence life-time experiment:}

Excitonic emission decay kinetics were measured using a time correlated single photon counting (TCSPC) module with a $405 \mathrm{~nm}$ picosecond pulsed semiconductor diode laser (EPL) of $51.1 \mathrm{ps}$ pulse width as an excitation source. The obtained decay profiles were best fitted with biexponential decay curve of which the resulted lifetimes (Table S3 and S4) were used for plotting the respective graphs (Figure 8, S13 and S10). Average fluorescence lifetime $(\mathrm{t})$ was calculated from their individual component $\tau_{1}$ and $\tau_{2}$ by using the equation: -

$$
\tau=\frac{A_{1} \tau_{1}+A_{2} \tau_{2}}{A_{1}+A_{2}}
$$

Where $\mathrm{A}_{1}$ and $\mathrm{A}_{2}$ pre exponential factors for $\tau_{1}$ and $\tau_{2}$ respectively.

Contribution of each individual component were calculated from $A_{1}$ and $A_{2}$ value as: -

$$
\begin{aligned}
& \% \text { of contribution of } \tau_{1}=\frac{A_{1}}{A_{1}+A_{2}} \times 100 \\
& \% \text { of contribution of } \tau_{2}=\frac{A_{2}}{A_{1}+A_{2}} \times 100
\end{aligned}
$$



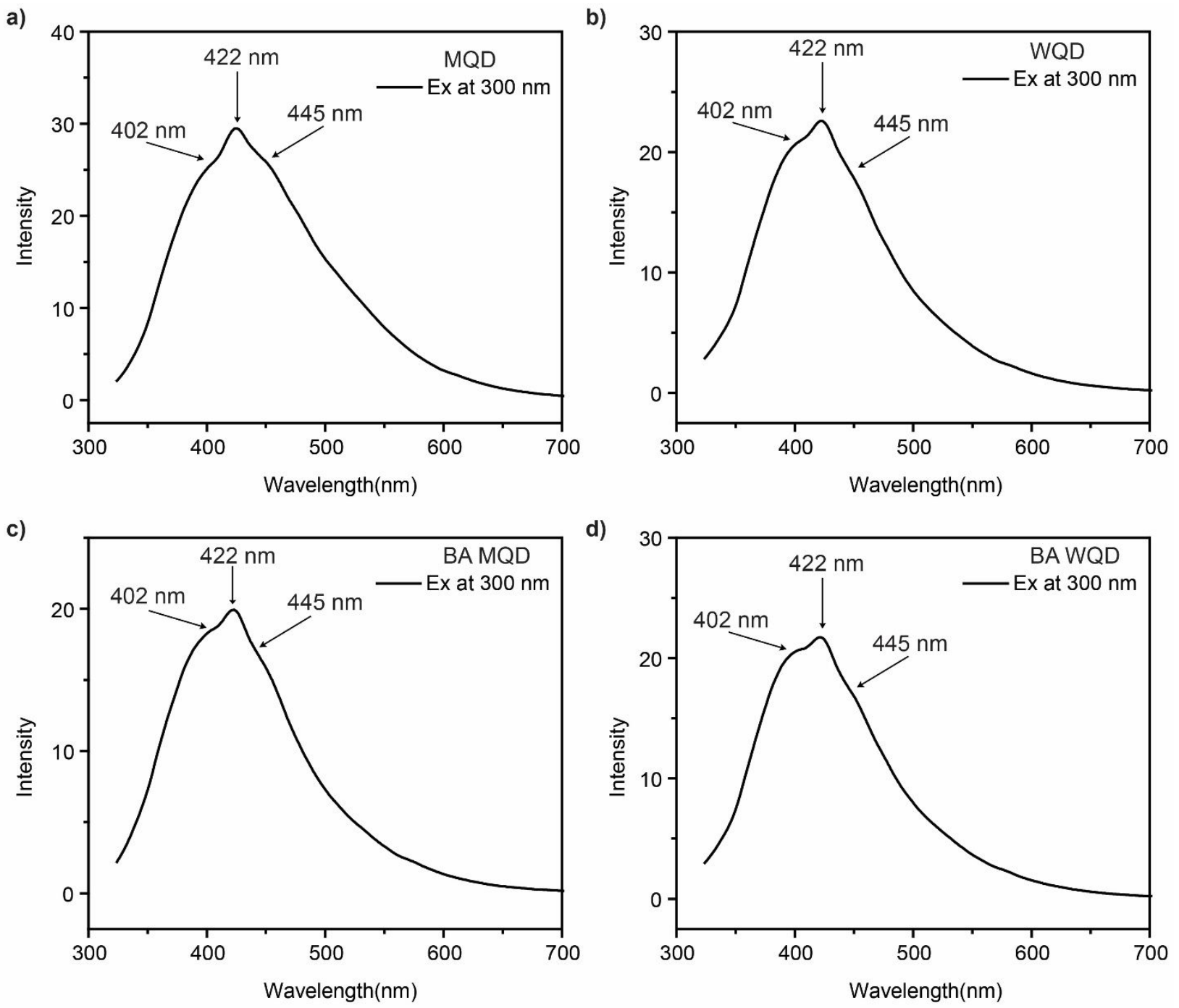

Figure S1 Fluorescence spectra of both native and functionalized quantum dots $\boldsymbol{\lambda}_{\max }=\mathbf{3 0 0}$

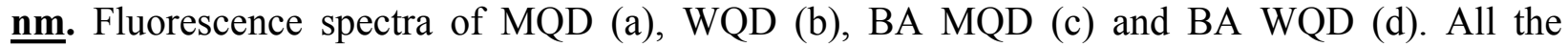
fluorescence spectra have two minor humps at $402 \mathrm{~nm}$ and $445 \mathrm{~nm}$ and maximum emission at 422 $\mathrm{nm}$. This indicates the polydispersity nature of quantum dots as well as excitation dependent emission. 
(In white light)

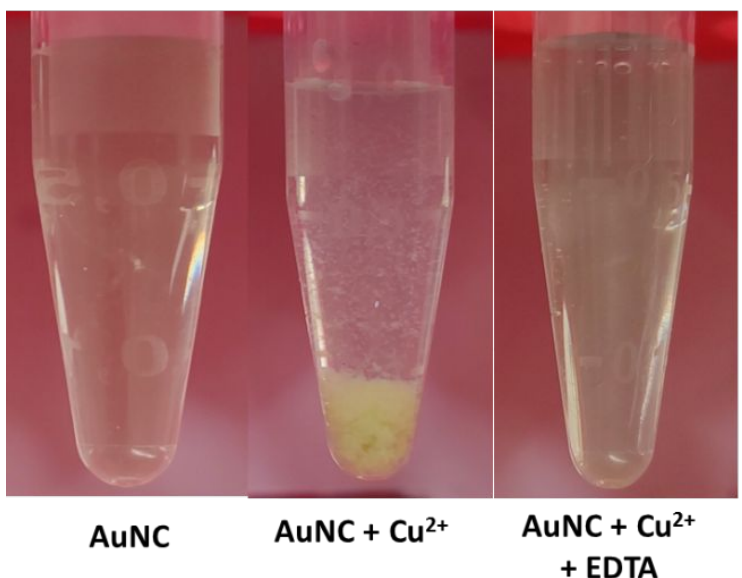

(In UV light)

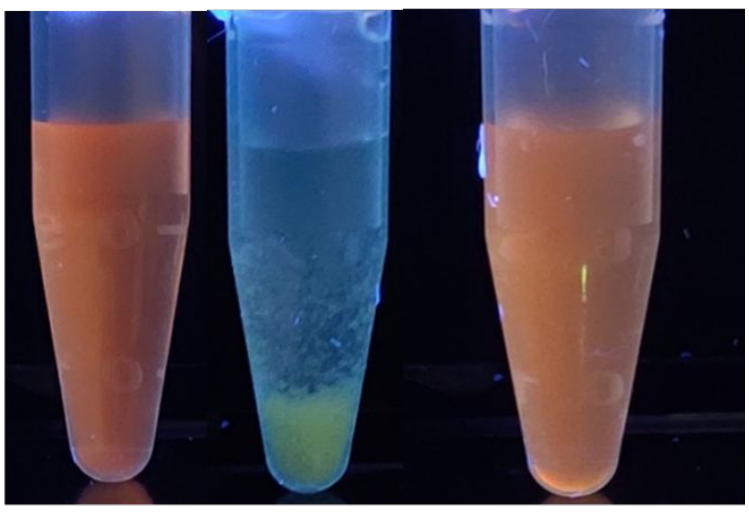

AuNC

$\mathrm{AuNC}+\mathrm{Cu}^{2+}$

$\mathrm{AuNC}+\mathrm{Cu}^{2+}$

+ EDTA

Figure S2 Naked eye visualization of Turn ON-OFF response for AuNC after addition of Cupric Chloride and EDTA disodium salt.

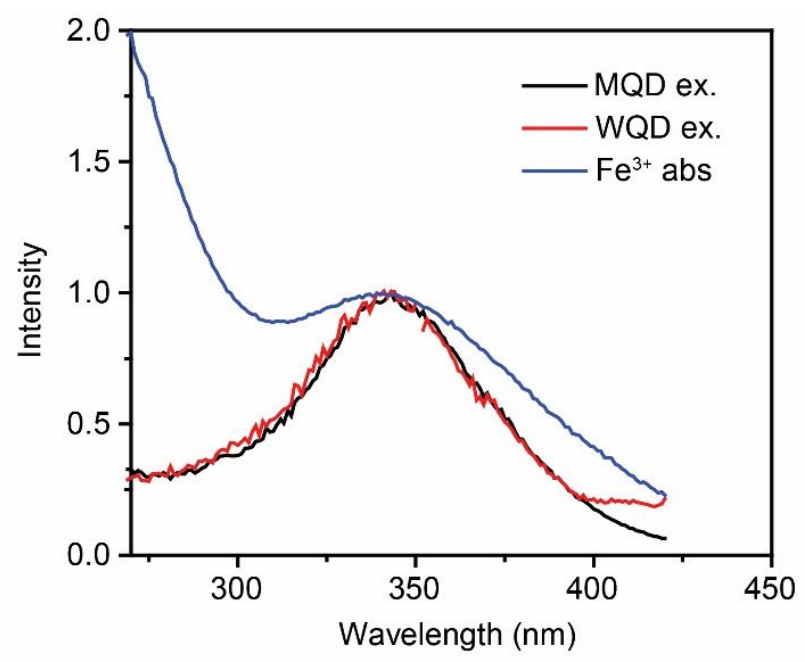

Figure S3 Primary inner-filter effect responsible for quenching of MQD and WQD with Fe $3^{+}$. Excitation spectra of MQD and WQD is overlapping completely with absorption spectra of aqueous solution of Ferric Chloride. 


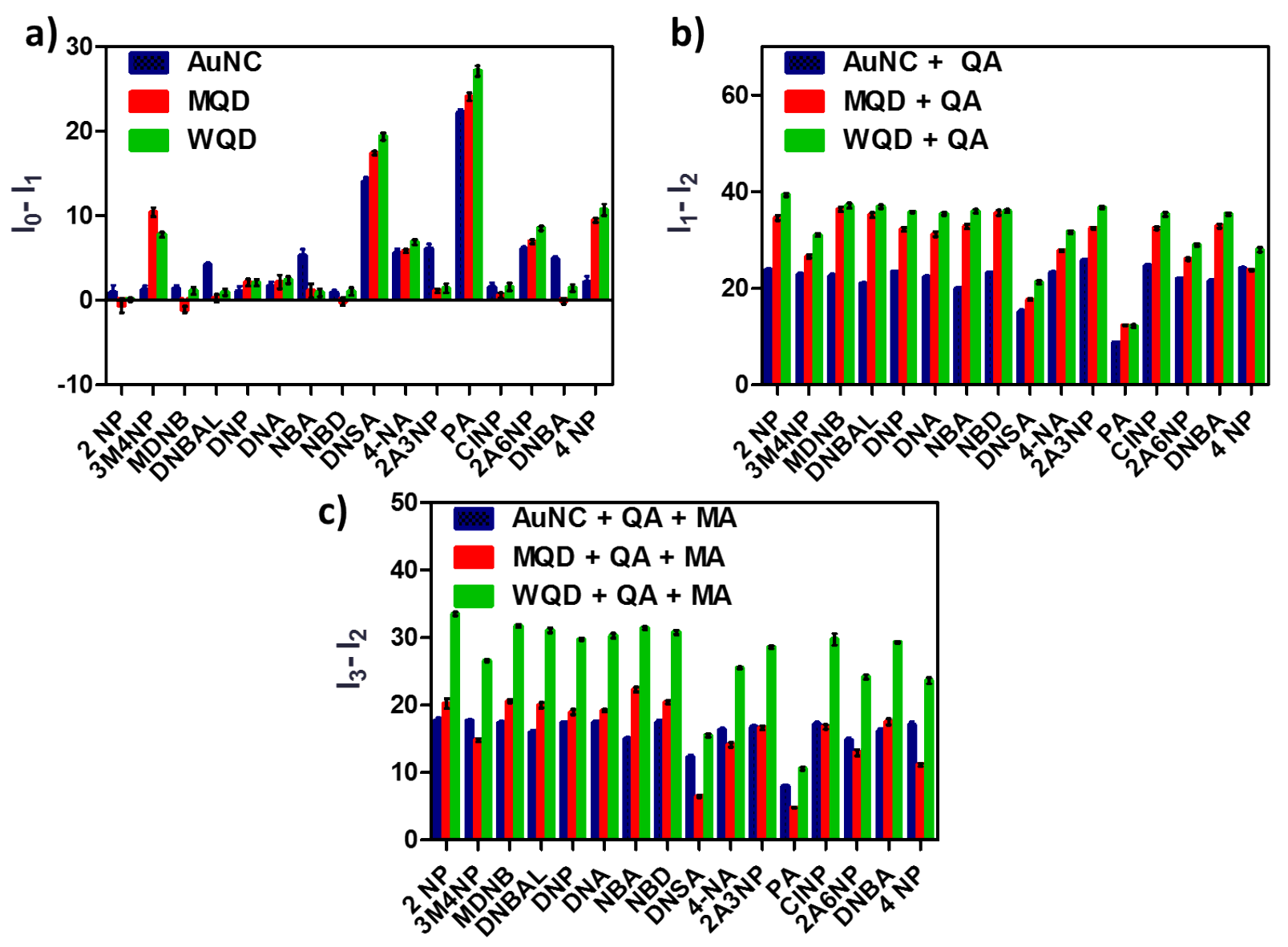

Figure S4 Fluorescence response pattern for native quantum dot array. Fluorescence response obtained for AuNC, MQD and WQD after addition of NACS (a), QA (b) and MA (c). 


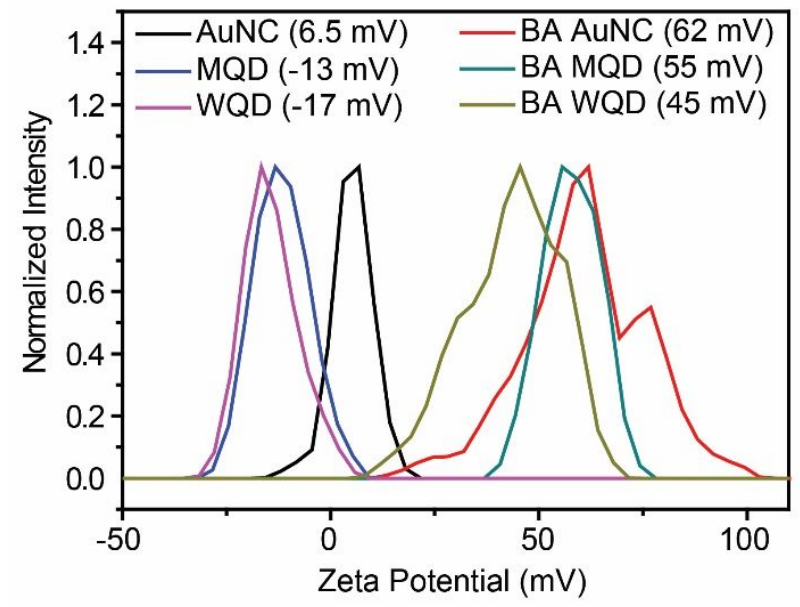

Figure S5. Zeta potential plot for native and functionalized quantum dots. AuNC, MQD and WQD are the native quantum dots with zeta potentials of $6.5 \mathrm{mV},-13 \mathrm{mV},-17 \mathrm{mV}$ respectively. BA AuNC, BA MQD and BA WQD are the thiol-functionalized quantum dots with zeta potentials of $62 \mathrm{mV}, 55 \mathrm{mV}$ and $45 \mathrm{mV}$.
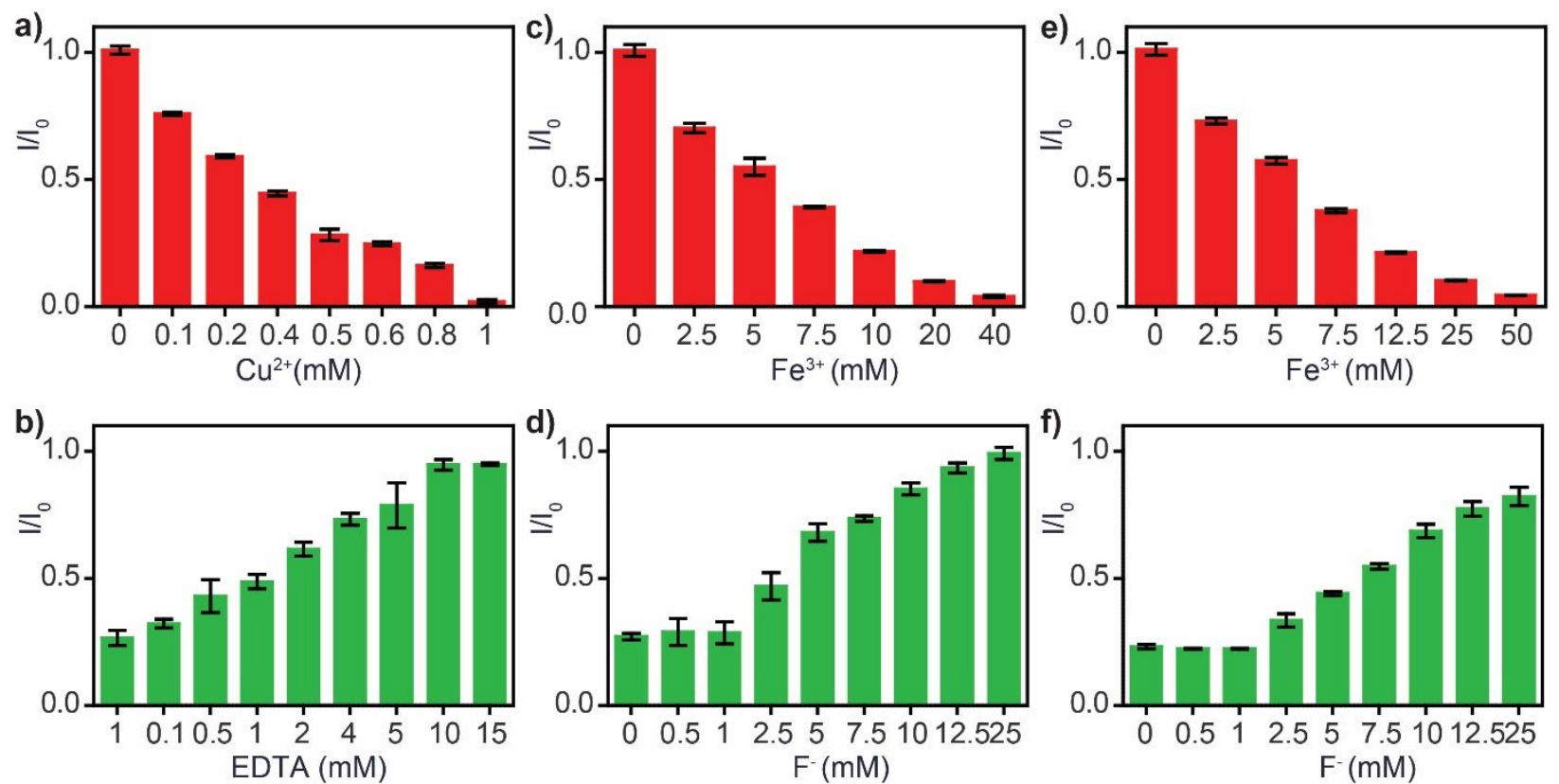

Figure S6 Sequential quenching and regeneration of fluorescence intensity of thiolfunctionalized quantum dots after addition of metal ions and corresponding masking agents. Fluorescence quenching of thiol-functionalized quantum dots after addition of metal ion $\mathrm{Cu}^{2+}$ to $B A A u N C s(a), \mathrm{Fe}^{3+}$ to BA MQD (c) and BA WQD (e) at increasing concentrations. Fluorescence signal regeneration after addition of masking agent EDTA to BA AuNCs $+C u^{2+}$ mixture (b), $F^{-}$to the mixture of $B A M Q D+F e^{3+}(d)$ and $B A W Q D+F e^{3+}(f)$. 
a)

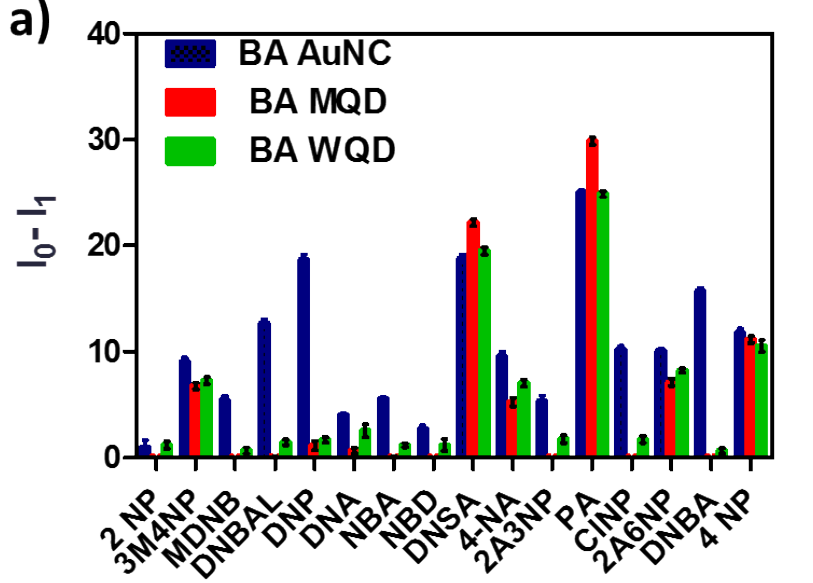

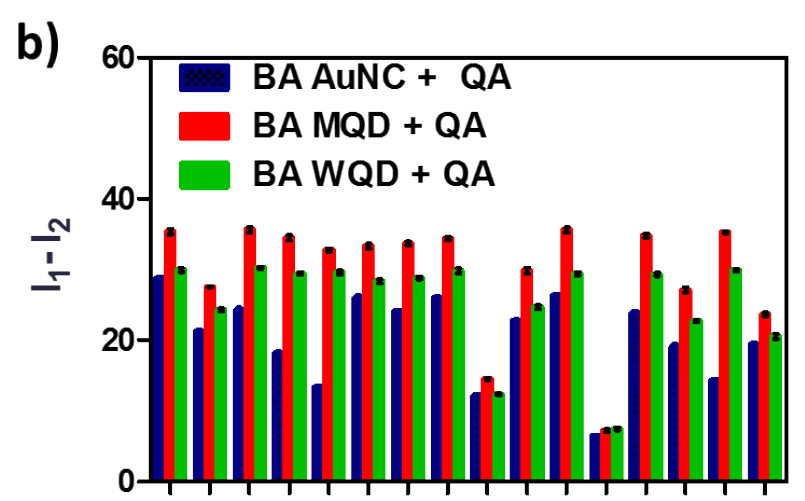

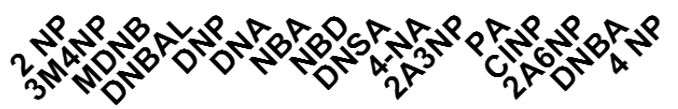

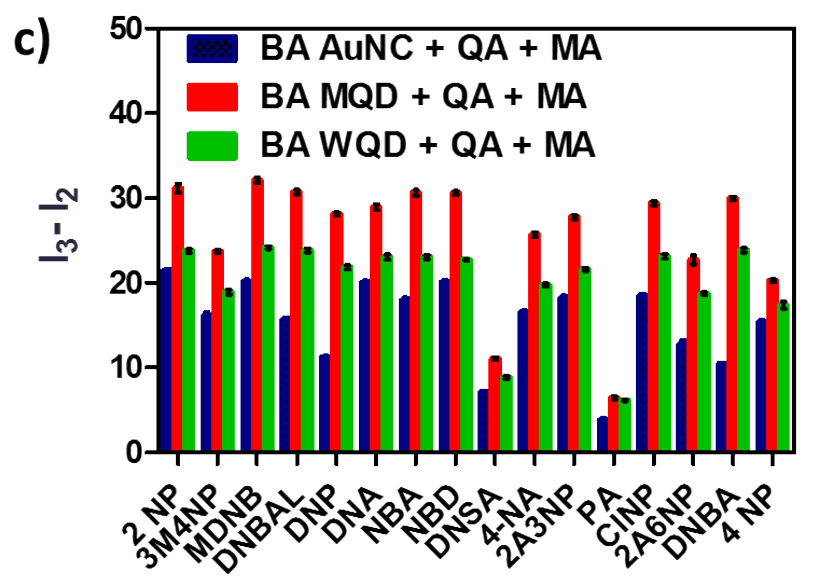

Figure S7 Fluorescence response pattern for functionalized quantum dot array. Fluorescence response obtained for BA AuNC, BA MQD and BA WQD after addition of NACS (a), QA (b) and MA (c). 

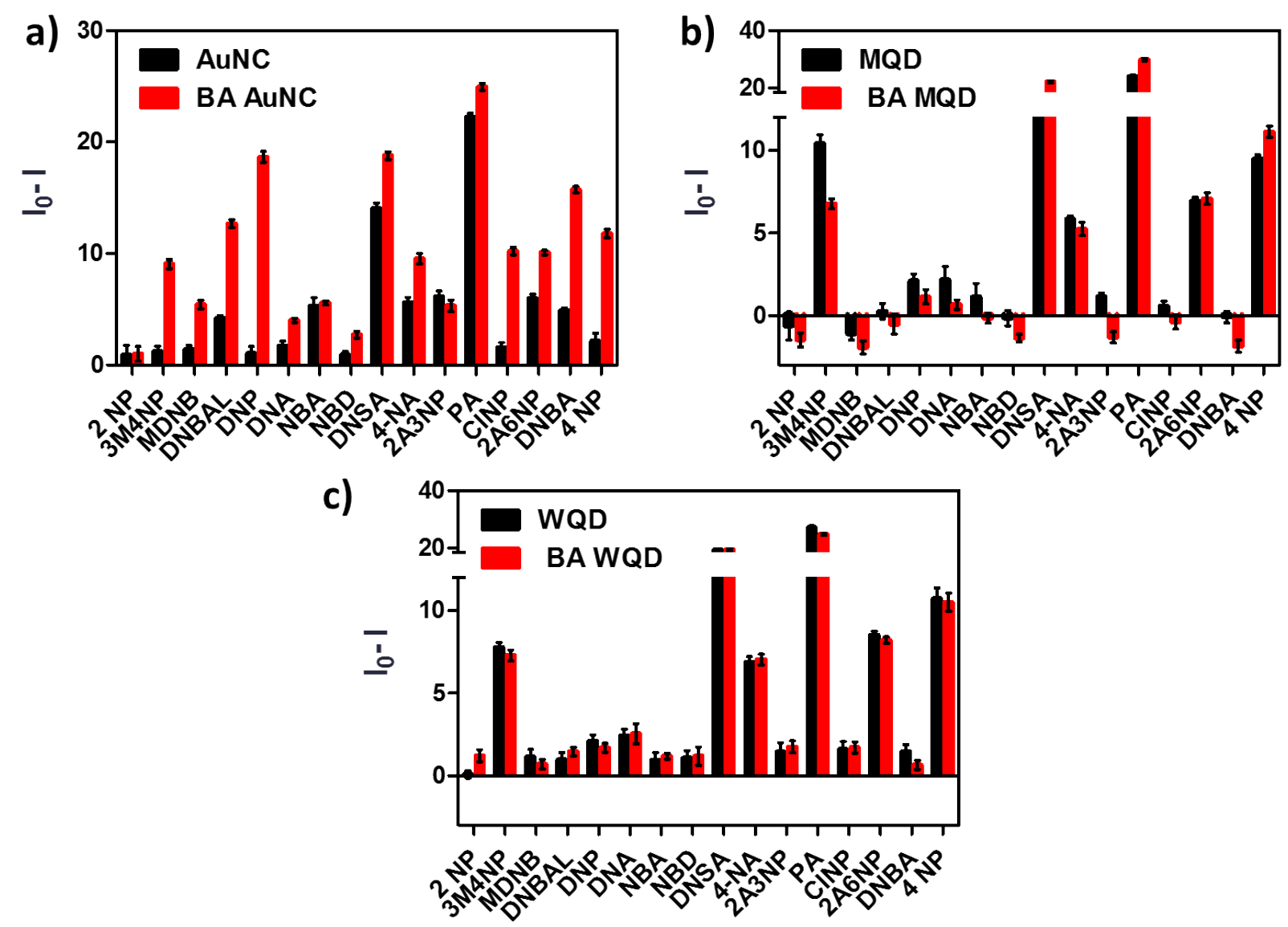

Figure S8 Comparison of fluorescence response pattern of native QDs with functionalized QDs array. Fluorescence response obtained after addition of different analytes to native and functionalized AuNC (a), MQD (b) and WQD (c).
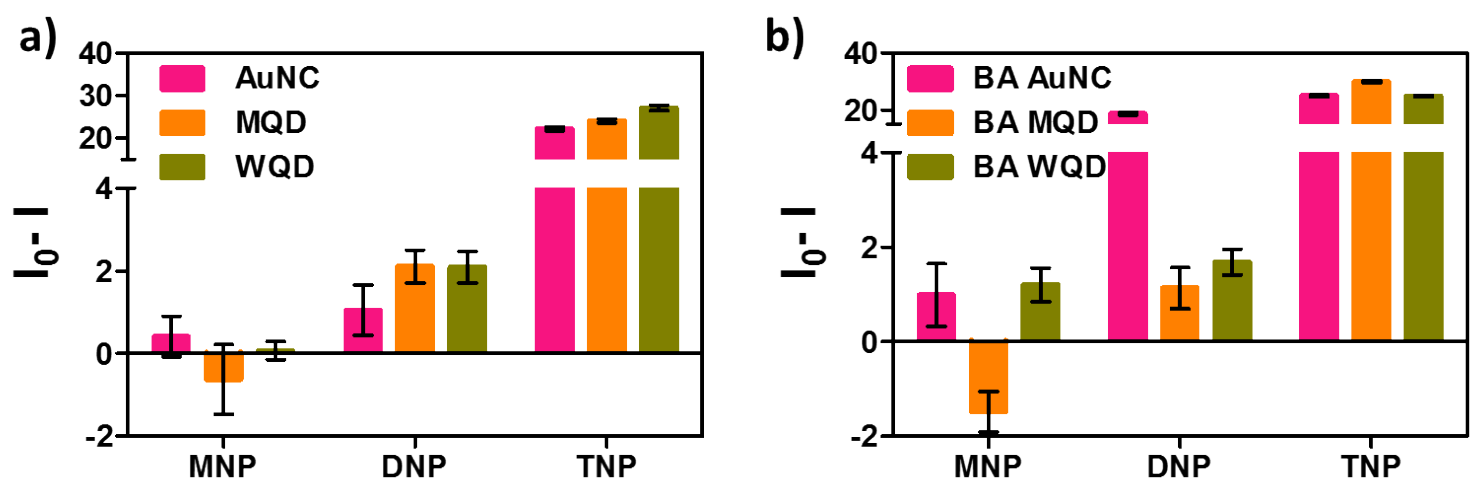

Figure S9 Fluorescence response pattern of native QDs and functionalized QDs array. Fluorescence response obtained after addition mono-, di- and tri-nitrophenol (abbreviated as MNP, DNP, TNP respectively) to native (a) and functionalized QDs (b). 

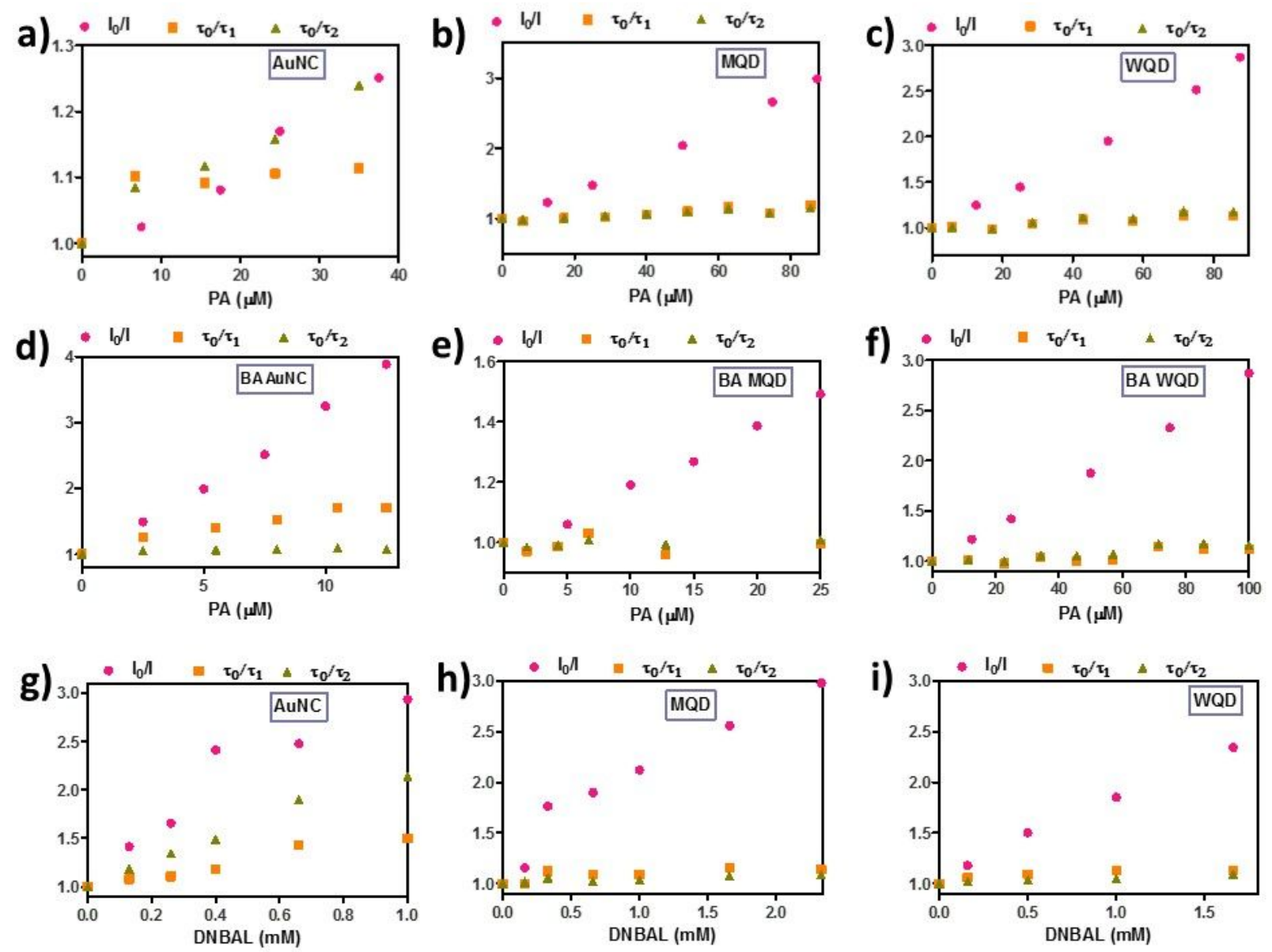

Figure S10 Plot of individual fluorescence lifetime component along with fluorescence intensity of all the quantum dots in different concentrations of PA and DNBAL. Change in fluorescence lifetime after addition of PA to AuNC (a), MQD (b), WQD(c), BA AuNC (d), BA MQD(e) and BA WQD (f). Change in fluorescence lifetime after addition of DNBAL to AuNC (g), MQD (h) and WQD(i). 

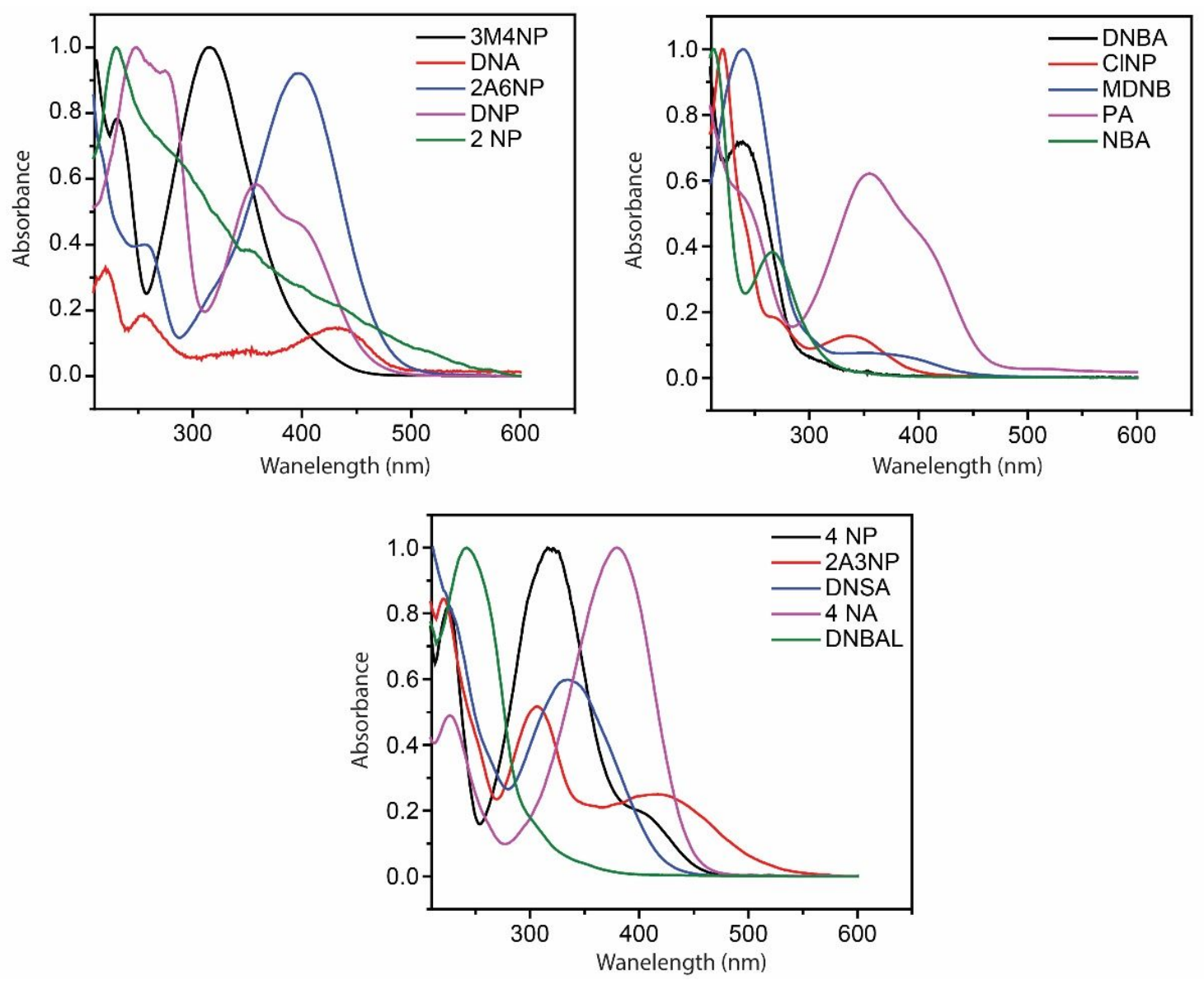

Figure S11 Absorption spectra of different nitro aromatic compounds. 

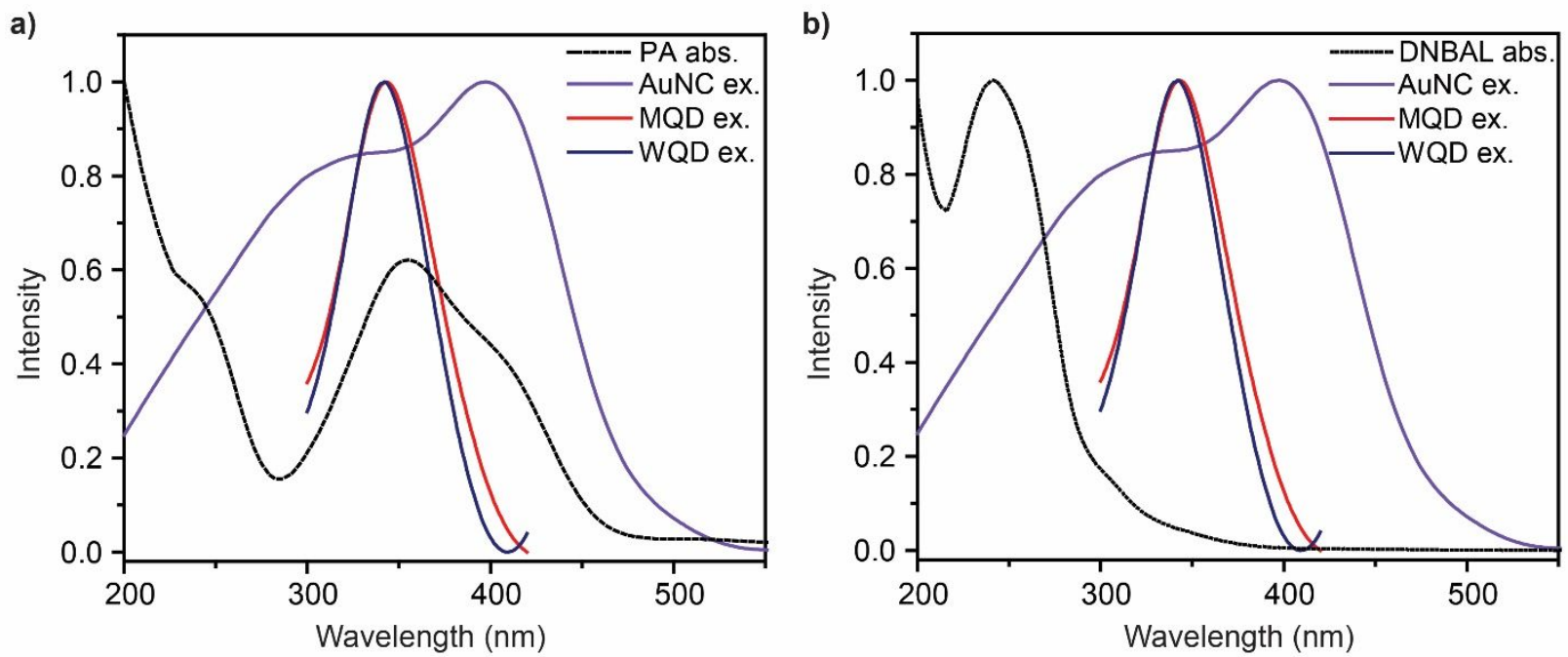

Figure S12 Optical study for confirmation of primary inner-filter effect. Excitation spectra of all three quantum dots along with picric acid i.e. PA (a) and dinitro benzaldehyde i.e. DNBAL (b). Absorption spectrum of PA (a) overlapped with excitation spectra of all three quantum dots which indicates the existence of primary inner-filter effect. In case of DNBAL (b), primary inner filter effect observed only in case of AuNC whose absorption spectra was in partially overlapping with AuNC excitation spectra.
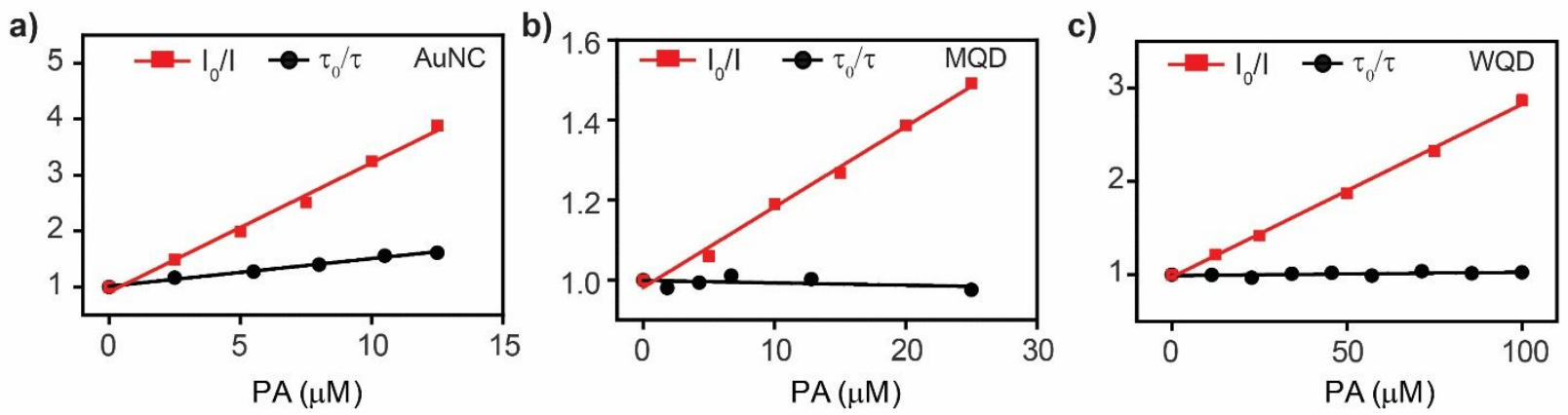

Figure S13 Fluorescence lifetime experiment of functionalized quantum dots in different concentrations of PA. Comparison plot of $\mathrm{I} / \mathrm{I}_{0}$ and $\tau / \tau_{0}$ for BA AuNCs (a), BA MQD (b)and BA WQD (c) in presence of different concentrations of PA. 

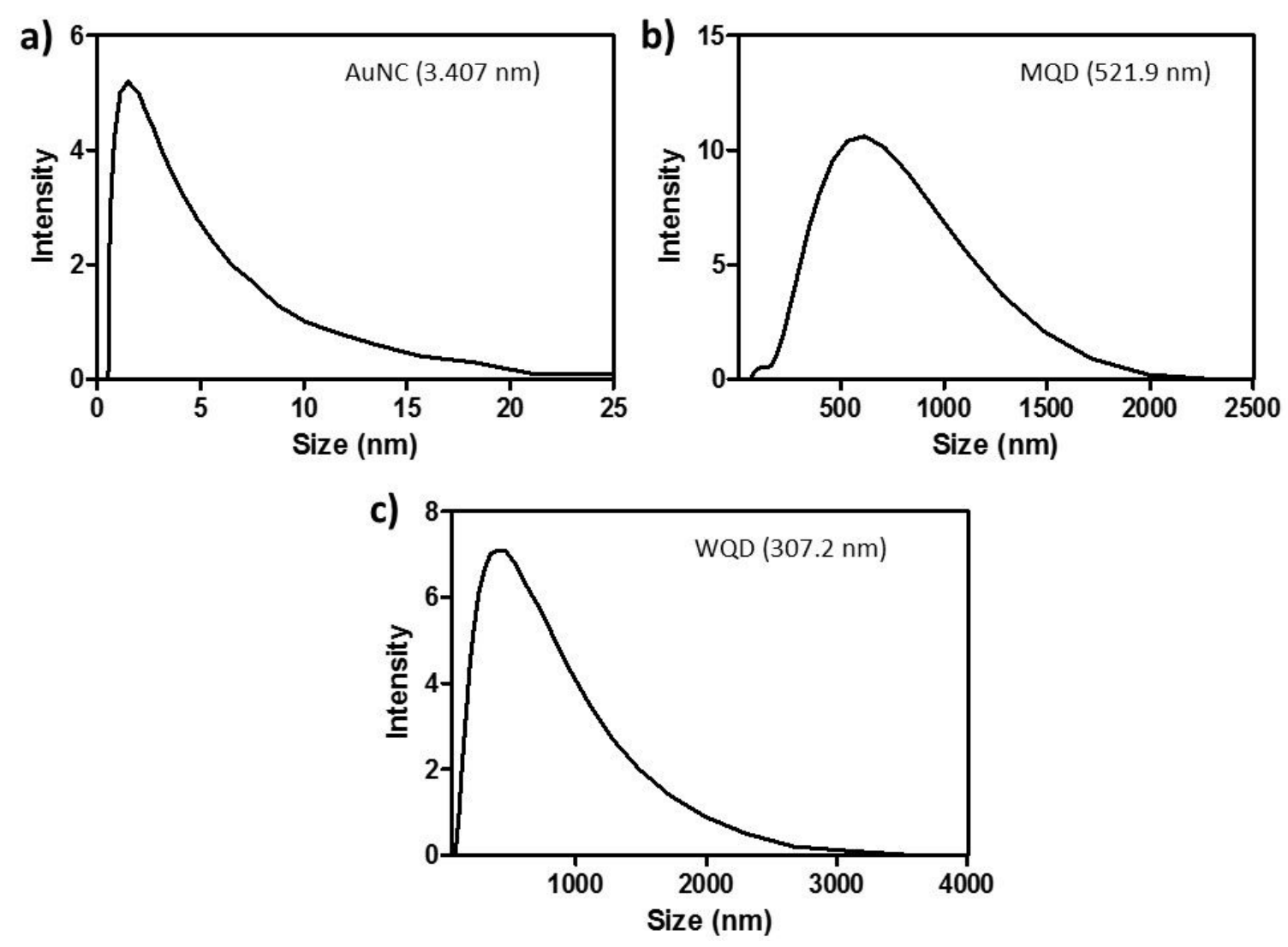

Figure S14 Size distribution plot of three different quantum dots. DLS measurement of a) AuNC (3.407 nm), b) MQD (521.9) and c) WQD (307.2 nm). In case of TMD QDs, high hydrodynamic size was observed due its hydrophilic surface with negative charges.

Table S1: Mahalanobis distances for different NACs by using native quantum dot array.

\begin{tabular}{|c|c|c|c|}
\hline NACs & 3 Channel & 6 Channel & 9 Channel \\
\hline 2 NP & -2.35 & -3032.265 & -4366.766 \\
\hline 3M4NP & -65.052 & -2551.931 & -3492.714 \\
\hline DNP & -5.991 & -2767.17 & -3841.528 \\
\hline NBA & -11.47 & -2739.571 & -3973.769 \\
\hline DNSA & -336.486 & -2228.291 & -2659.839 \\
\hline 4-NA & -44.905 & -2497.439 & -3321.545 \\
\hline 2A3NP & -14.686 & -2810.671 & -3762.716 \\
\hline PA & -689.861 & -2155.809 & -2494.963 \\
\hline 4 NP & -80.921 & -2262.853 & -3022.984 \\
\hline
\end{tabular}


Table S2: Mahalanobis distances for different NACs by using thiol-functionalized quantum dot array.

\begin{tabular}{|c|c|c|c|}
\hline NACs & 3 Channel & 6 Channel & 9 Channel \\
\hline 2 NP & -4.876 & -4943.14 & -5433.45 \\
\hline MDNB & -17.987 & -4703.574 & -5220.561 \\
\hline DNBAL & -84.001 & -4376.328 & -5062.719 \\
\hline DNP & -184.445 & -4218.476 & -5120.861 \\
\hline DNA & -14.805 & -4576.878 & -5056.526 \\
\hline DNSA & -730.085 & -3124.878 & -3873.491 \\
\hline 4-NA & -98.386 & -4258.233 & -4838.615 \\
\hline 2A3NP & -18.138 & -4861.562 & -5520.236 \\
\hline PA & -1269.786 & -3047.616 & -3811.71 \\
\hline CINP & -56.047 & -4761.681 & -5390.553 \\
\hline 2A6NP & -129.866 & -3704.823 & -4337.448 \\
\hline DNBA & -125.903 & -4231.224 & -5216.267 \\
\hline 4 NP & -222.701 & -3618.832 & -4066.043 \\
\hline
\end{tabular}

Table S3: Different fluorescence lifetime decay component for three different quantum dots against two quenchers i.e. PA and DNBAL at different concentrations.

\begin{tabular}{|c|c|c|c|c|c|c|}
\hline $\begin{array}{c}\text { Quantum } \\
\text { dots }\end{array}$ & Quenchers & $\begin{array}{c}\text { Concentration } \\
\text { of Quencher }\end{array}$ & $\boldsymbol{\tau}_{\mathbf{1}}$ & $\boldsymbol{\tau}_{\mathbf{2}}$ & $\mathbf{A 1}$ & $\mathbf{A 2}$ \\
\hline AuNC & PA & 0 & 314.54 & 2145.6 & 1046.76 & 546.58 \\
\hline AuNC & PA & 6.7165 & 287.74 & 1983.3 & 836.25 & 414.97 \\
\hline AuNC & PA & 15.554 & 290.94 & 1926.1 & 643.77 & 317.21 \\
\hline AuNC & PA & 24.392 & 286.76 & 1858.2 & 521.76 & 250.38 \\
\hline AuNC & PA & 35 & 284.22 & 1734.4 & 528.34 & 253.6 \\
\hline MQD & PA & 0 & 2.08 & 7.5 & 3727 & 1357 \\
\hline MQD & PA & 5.714 & 2.17 & 7.63 & 2078 & 726 \\
\hline MQD & PA & 17.142 & 2.06 & 7.44 & 1886 & 678 \\
\hline MQD & PA & 28.59 & 2.04 & 7.2 & 1250 & 509 \\
\hline MQD & PA & 40 & 1.96 & 7.02 & 1042 & 416 \\
\hline MQD & PA & 51.426 & 1.88 & 6.82 & 717 & 323 \\
\hline MQD & PA & 62.854 & 1.78 & 6.55 & 584 & 276 \\
\hline MQD & PA & 74.282 & 1.94 & 6.96 & 875 & 355 \\
\hline MQD & PA & 85.7 & 1.75 & 6.52 & 464 & 220 \\
\hline
\end{tabular}




\begin{tabular}{|c|c|c|c|c|c|c|} 
WQD & PA & 0 & 2.17 & 7.64 & 4389 & 1003 \\
\hline WQD & PA & 5.714 & 2.15 & 7.58 & 2811 & 649 \\
\hline WQD & PA & 17.142 & 2.21 & 7.74 & 2314 & 480 \\
\hline WQD & PA & 28.59 & 2.08 & 7.22 & 1624 & 415 \\
\hline WQD & PA & 42.85 & 1.98 & 6.86 & 1223 & 346 \\
\hline WQD & PA & 57.14 & 2.03 & 6.93 & 1034 & 300 \\
\hline WQD & PA & 71.425 & 1.9 & 6.45 & 724 & 251 \\
\hline WQD & PA & 85.7 & 1.91 & 6.5 & 601 & 202 \\
\hline BA AuNC & PA & 0 & 353.32 & 1868.4 & 1618.73 & 928.19 \\
\hline BA AuNC & PA & 2.5 & 280.83 & 1785.9 & 1243.37 & 609.8 \\
\hline BA AuNC & PA & 5.5 & 253.54 & 1756.1 & 976.94 & 426.29 \\
\hline BA AuNC & PA & 8 & 231.22 & 1745.9 & 843.92 & 318.78 \\
\hline BA AuNC & PA & 10.5 & 208.1 & 1710.8 & 723.59 & 237.6 \\
\hline BA AuNC & PA & 12.5 & 207.66 & 1739.92 & 603.82 & 180.64 \\
\hline BA MQD & PA & 0 & 2.01 & 7.28 & 4804 & 1777 \\
\hline BA MQD & PA & 1.829 & 2.07 & 7.39 & 3737 & 1377 \\
\hline BA MQD & PA & 4.268 & 2.04 & 7.35 & 3263 & 1185 \\
\hline BA MQD & PA & 6.707 & 1.95 & 7.22 & 2297 & 868 \\
\hline BA MQD & PA & 12.8045 & 2.09 & 7.33 & 1736 & 594 \\
\hline BA MQD & PA & 25 & 2.02 & 7.23 & 1370 & 553 \\
\hline BA WQD & PA & 0 & 2.06 & 8.16 & 3184 & 879 \\
\hline BA WQD & PA & 11.45 & 2.04 & 8.03 & 1781 & 514 \\
\hline BA WQD & PA & 22.856 & 2.11 & 8.15 & 1340 & 398 \\
\hline BA WQD & PA & 34.284 & 1.98 & 7.74 & 1089 & 338 \\
\hline BA WQD & PA & 45.712 & 2.05 & 7.76 & 887 & 252 \\
\hline BA WQD & PA & 57.14 & 2.03 & 7.62 & 633 & 207 \\
\hline BA WQD & PA & 71.425 & 1.8 & 6.98 & 615 & 240 \\
\hline BA WQD & PA & 85.7 & 1.83 & 6.95 & 471 & 195 \\
\hline BA WQD & PA & 100 & 1.85 & 7.04 & 525 & 203 \\
\hline AuNC & DNBAL & 0 & 282.58 & 1807.3 & 522 & 215.62 \\
\hline AuNC & DNBAL & 0.13 & 261.41 & 1525.78 & 467.94 & 186.55 \\
\hline AuNC & DNBAL & 0.26 & 255.18 & 1344.88 & 514.73 & 208.03 \\
\hline AuNC & DNBAL & 0.4 & 239.88 & 1210.24 & 667.67 & 278.43 \\
\hline AuNC & DNBAL & 0.66 & 197.12 & 951.45 & 515.76 & 250.71 \\
\hline AuNC & DNBAL & 1 & 188.17 & 842.98 & 547.29 & 266.83 \\
\hline MQD & DNBAL & 0 & 2.54 & 10.16 & 1636.85 & 608.92 \\
\hline MQD & DNBAL & 0.16 & 2.55 & 9.99 & 1787.98 & 674.93 \\
\hline MQD & DNBAL & 0.33 & 2.26 & 9.61 & 1215.6 & 485.6 \\
\hline MQD & DNBAL & 0.66 & 2.33 & 9.93 & 1569.1 & 580 \\
\hline
\end{tabular}




\begin{tabular}{|c|c|c|c|c|c|c|} 
MQD & DNBAL & 1 & 2.33 & 9.78 & 933.7 & 366.26 \\
\hline MQD & DNBAL & 1.66 & 2.19 & 9.44 & 883.9 & 336.36 \\
\hline MQD & DNBAL & 2.33 & 2.23 & 9.3 & 714.84 & 294.46 \\
\hline WQD & DNBAL & 0 & 2.48 & 10.63 & 976.24 & 277.07 \\
\hline WQD & DNBAL & 0.16 & 2.34 & 10.4 & 985.55 & 308.79 \\
\hline WQD & DNBAL & 0.5 & 2.27 & 10.23 & 807.85 & 251.03 \\
\hline WQD & DNBAL & 1 & 2.2 & 10.09 & 839.76 & 258.86 \\
\hline WQD & DNBAL & 1.66 & 2.2 & 9.71 & 617.12 & 214.54 \\
\hline
\end{tabular}

Table S4: Dataset for fluorescence response pattern by using native quantum dot array.

\begin{tabular}{|c|c|c|c|c|c|c|c|c|c|}
\hline NACs & AuNC & $\begin{array}{l}\text { AuNC } \\
+ \text { QA }\end{array}$ & $\begin{array}{c}\text { AuNC } \\
+\mathrm{QA}+\mathrm{MA} \\
\end{array}$ & MQD & $\begin{array}{l}\text { MQD } \\
+ \text { QA } \\
\end{array}$ & $\begin{array}{c}M Q D \\
+Q A+M A \\
\end{array}$ & WQD & $\begin{array}{l}\text { WQD } \\
+ \text { QA }\end{array}$ & $\begin{array}{c}\text { WQD } \\
+Q A+M A \\
\end{array}$ \\
\hline $2 N P$ & 0.5176 & 30.22218 & 22.44198 & -0.8325 & 35.151 & 22.9934 & 0.2117 & 39.28278 & 34.32698 \\
\hline $2 N P$ & 1.708 & 28.43577 & 20.54407 & 0.0901 & 34.53372 & 21.42582 & -0.2432 & 40.81317 & 33.80267 \\
\hline $2 \mathrm{NP}$ & 1.68 & 26.69947 & 22.20957 & 3.1034 & 33.87091 & 19.12291 & -0.059 & 38.55054 & 32.79574 \\
\hline $2 \mathrm{NP}$ & -1.4122 & 31.61255 & 23.83985 & -1.7317 & 36.85251 & 19.11581 & 0.8478 & 38.8939 & 33.5099 \\
\hline $2 \mathrm{NP}$ & -0.3743 & 29.33702 & 22.49872 & -1.5676 & 33.14193 & 18.24703 & 0.4123 & 38.64303 & 32.68883 \\
\hline $2 N P$ & 0.373 & 30.55078 & 22.18208 & -2.8046 & 33.83812 & 20.45802 & -0.7155 & 39.78533 & 33.87063 \\
\hline $3 M 4 N P$ & 0.9598 & 28.2733 & 21.2231 & 8.9132 & 27.30196 & 15.39086 & 6.7279 & 32.26131 & 26.77111 \\
\hline 3M4NP & -0.7292 & 28.5496 & 21.4474 & 9.9584 & 27.20103 & 15.25323 & 7.8638 & 30.93371 & 27.12691 \\
\hline 3M4NP & 2.8241 & 26.49951 & 19.62871 & 9.9996 & 26.13764 & 15.03234 & 8.4764 & 30.01515 & 26.13785 \\
\hline 3M4NP & 1.4757 & 28.14277 & 20.87187 & 12.6411 & 25.25276 & 14.48906 & 7.0464 & 30.74993 & 26.30753 \\
\hline 3M4NP & 2.0925 & 29.05365 & 21.08925 & 10.5983 & 26.30287 & 13.92747 & 8.0656 & 30.89339 & 26.31559 \\
\hline 3M4NP & 0.6774 & 27.71651 & 21.62641 & 10.4845 & 27.24331 & 14.72361 & 8.4683 & 31.3022 & 26.6837 \\
\hline DNP & -0.6389 & 28.63719 & 21.92289 & 2.3422 & 33.89952 & 20.33142 & 2.5763 & 36.07572 & 28.86862 \\
\hline DNP & 1.3933 & 29.06015 & 19.77985 & 2.7458 & 31.58868 & 19.49478 & 1.1297 & 36.31458 & 30.01268 \\
\hline DNP & 2.2458 & 28.63739 & 22.48219 & 1.9488 & 31.89355 & 18.36985 & 0.6594 & 36.06186 & 29.45786 \\
\hline DNP & 2.8323 & 28.52312 & 21.08692 & 0.26 & 32.04583 & 19.48433 & 2.7883 & 35.20218 & 29.93898 \\
\hline DNP & -0.8227 & 30.40285 & 21.96955 & 3.0103 & 31.33834 & 17.97424 & 2.8761 & 35.25608 & 29.99708 \\
\hline DNP & 1.276 & 28.72052 & 22.43792 & 2.3555 & 32.42859 & 18.00339 & 2.4905 & 35.79877 & 30.21507 \\
\hline NBA & 5.485 & 23.37988 & 18.01458 & 2.7729 & 31.50935 & 23.19005 & -0.9013 & 37.62262 & 31.58852 \\
\hline NBA & 3.8048 & 24.5189 & 17.3518 & 2.1224 & 32.4285 & 22.7138 & 2.6792 & 35.1401 & 31.0984 \\
\hline NBA & 3.2285 & 25.48771 & 17.63011 & 3.4984 & 31.95628 & 20.84278 & 1.0115 & 36.12906 & 32.14486 \\
\hline NBA & 6.4287 & 21.83641 & 18.05141 & 0.2582 & 32.60092 & 23.15032 & 1.2476 & 35.59164 & 31.80144 \\
\hline
\end{tabular}




\begin{tabular}{|c|c|c|c|c|c|c|c|c|c|}
\hline NBA & 4.5428 & 25.53497 & 18.87777 & -0.1238 & 34.16014 & 22.14884 & 0.7225 & 36.40816 & 31.28596 \\
\hline NBA & 8.2102 & 23.05224 & 20.27664 & -1.716 & 34.17066 & 21.72256 & 0.7991 & 34.9226 & 30.5871 \\
\hline DNSA & 14.8254 & 15.21695 & 13.84175 & 16.9208 & 17.01652 & 5.86752 & 17.9935 & 22.42912 & 16.03282 \\
\hline DNSA & 12.1556 & 18.82762 & 15.77162 & 18.292 & 17.71239 & 6.66379 & 20.7137 & 21.77596 & 15.62656 \\
\hline DNSA & 14.8911 & 14.3575 & 14.5454 & 16.9064 & 18.26779 & 6.93849 & 19.338 & 21.42031 & 14.58591 \\
\hline DNSA & 15.4273 & 16.30547 & 12.45077 & 18.0423 & 17.70061 & 6.65701 & 18.3782 & 20.93673 & 16.04843 \\
\hline DNSA & 13.1261 & 15.99423 & 13.10303 & 17.2734 & 17.38635 & 6.57075 & 20.2863 & 19.51028 & 15.93678 \\
\hline DNSA & 13.7589 & 15.9633 & 14.6245 & 17.0541 & 17.94462 & 6.22892 & 19.5433 & 21.34836 & 14.70536 \\
\hline 4-NA & 5.7679 & 23.78741 & 18.14141 & 6.4819 & 27.51908 & 13.20288 & 6.5861 & 31.8347 & 25.5385 \\
\hline 4-NA & 7.5033 & 23.06061 & 18.62681 & 5.0872 & 27.09452 & 13.23692 & 7.1007 & 32.63279 & 25.75239 \\
\hline 4-NA & 6.0473 & 24.86019 & 18.57839 & 5.7983 & 28.55173 & 15.06963 & 7.8529 & 31.80136 & 24.79816 \\
\hline 4-NA & 3.9694 & 25.19687 & 18.22657 & 6.177 & 27.23499 & 13.56999 & 5.5831 & 30.95734 & 25.52454 \\
\hline 4-NA & 5.1449 & 25.28668 & 18.31238 & 5.5107 & 28.37597 & 15.15347 & 6.5596 & 31.58824 & 25.64674 \\
\hline 4-NA & 5.105 & 24.22639 & 19.43209 & 5.9906 & 28.02512 & 14.49112 & 7.5743 & 31.00379 & 25.85509 \\
\hline $2 A 3 N P$ & 6.5452 & 25.25069 & 19.07209 & 0.6734 & 32.1003 & 16.3226 & 2.4574 & 36.47427 & 28.73597 \\
\hline 2A3NP & 4.1096 & 24.1926 & 18.5301 & 0.9084 & 32.11111 & 16.33061 & 2.2982 & 36.34382 & 28.15712 \\
\hline 2A3NP & 5.7262 & 24.84807 & 18.27577 & 1.2447 & 32.71404 & 17.48104 & -0.1528 & 37.3495 & 28.12 \\
\hline $2 \mathrm{~A} 3 \mathrm{NP}$ & 5.6183 & 22.55818 & 16.03428 & 2.0121 & 32.02587 & 15.97187 & 0.7566 & 36.69982 & 28.78152 \\
\hline 2A3NP & 6.9877 & 24.15039 & 17.97099 & 0.5369 & 33.36155 & 16.31835 & 0.1074 & 37.46787 & 29.33517 \\
\hline 2A3NP & 7.7793 & 22.49433 & 18.68093 & 1.3943 & 32.32484 & 17.36284 & 3.1539 & 35.89968 & 28.21238 \\
\hline PA & 23.2857 & 8.73307 & 8.34037 & 22.8941 & 12.54558 & 4.75223 & 26.4979 & 12.66978 & 11.21338 \\
\hline $\mathrm{PA}$ & 23.1933 & 8.23072 & 7.89302 & 23.11 & 12.71215 & 4.83884 & 25.4157 & 13.40165 & 11.02245 \\
\hline PA & 21.733 & 8.77602 & 7.78949 & 24.5857 & 12.48029 & 5.01708 & 26.2646 & 12.46287 & 11.26867 \\
\hline PA & 21.29725 & 7.59996 & 7.70186 & 24.0344 & 11.8085 & 4.42861 & 28.0114 & 11.57056 & 9.92336 \\
\hline PA & 22.5507 & 8.29163 & 7.63464 & 23.8268 & 12.32711 & 5.00374 & 29.6949 & 11.59252 & 10.34842 \\
\hline $\mathrm{PA}$ & 21.1481 & 8.728 & 7.69455 & 26.0918 & 11.97331 & 4.63144 & 26.9916 & 11.43897 & 9.88037 \\
\hline $4 \mathrm{NP}$ & -1.1004 & 27.7593 & 19.3387 & 8.8113 & 24.11749 & 10.82709 & 8.3044 & 29.65719 & 25.28649 \\
\hline $4 \mathrm{NP}$ & 2.9163 & 27.54799 & 20.85899 & 8.9696 & 23.40707 & 10.24967 & 12.2876 & 28.52429 & 23.90629 \\
\hline $4 \mathrm{NP}$ & 3.0553 & 27.30643 & 20.68473 & 9.0249 & 24.18506 & 11.24786 & 9.71 & 27.47505 & 22.10855 \\
\hline $4 \mathrm{NP}$ & 1.2945 & 26.73786 & 22.19766 & 10.3722 & 23.70359 & 11.74209 & 10.1362 & 28.90794 & 23.43174 \\
\hline $4 \mathrm{NP}$ & 2.8808 & 25.99985 & 19.23075 & 10.0292 & 23.90848 & 11.76338 & 12.4875 & 26.42843 & 22.79273 \\
\hline $4 \mathrm{NP}$ & 3.7352 & 25.36752 & 19.92492 & 9.7043 & 23.13594 & 10.96164 & 11.403 & 26.92546 & 24.12656 \\
\hline
\end{tabular}

Table S5: Dataset for fluorescence response pattern by using thiol-functionalized quantum dot array.

\begin{tabular}{|c|c|c|c|c|c|c|c|c|c|}
\hline NACs & BA AuNC & $\begin{array}{c}\text { BA AuNC } \\
+ \text { QA }\end{array}$ & $\begin{array}{c}\text { BA AuNC } \\
+ \text { QA + MA }\end{array}$ & BA MQD & $\begin{array}{c}\text { BA MQD } \\
\text { + QA }\end{array}$ & $\begin{array}{c}\text { BA MQD } \\
+ \text { QA + MA }\end{array}$ & BA WQD & $\begin{array}{c}\text { BA WQD } \\
+ \text { QA }\end{array}$ & $\begin{array}{c}\text { BA WQD } \\
+ \text { QA + MA }\end{array}$ \\
\hline $2 \mathrm{NP}$ & 1.0934 & 28.42341 & 21.04121 & -1.1992 & 33.76118 & 29.29148 & -0.3579 & 30.54291 & 23.62111 \\
\hline $2 \mathrm{NP}$ & 3.1501 & 27.59734 & 21.01424 & -3.3801 & 36.20403 & 30.22623 & 1.099 & 29.98469 & 24.37359 \\
\hline
\end{tabular}




\begin{tabular}{|c|c|c|c|c|c|c|c|c|c|}
\hline $2 \mathrm{NP}$ & 2.6874 & 28.39606 & 22.22506 & -1.1622 & 35.07101 & 32.61751 & 2.1744 & 30.61904 & 24.34714 \\
\hline $2 \mathrm{NP}$ & 0.0958 & 28.9284 & 21.773 & -0.5628 & 36.32732 & 31.57202 & 1.4951 & 30.45851 & 24.16901 \\
\hline $2 N P$ & -0.8211 & 29.87307 & 20.67277 & -0.6868 & 34.79879 & 30.68509 & 1.0267 & 28.93387 & 23.17197 \\
\hline $2 \mathrm{NP}$ & -0.2518 & 29.37834 & 21.84424 & -1.8727 & 36.06604 & 32.50894 & 1.7978 & 29.19479 & 23.17699 \\
\hline MDNB & 4.9477 & 23.78541 & 18.97021 & -1.2455 & 35.70247 & 31.31207 & 1.2611 & 30.02399 & 24.71099 \\
\hline MDNB & 6.4761 & 24.50186 & 20.25416 & -2.2684 & 34.86258 & 32.03298 & 0.9216 & 29.89898 & 24.01258 \\
\hline MDNB & 5.8007 & 25.00321 & 20.82891 & -3.2082 & 34.80101 & 31.69631 & 0.5866 & 30.66103 & 23.53413 \\
\hline MDNB & 5.2277 & 24.43922 & 20.12922 & -0.9421 & 35.02004 & 33.48444 & 1.5853 & 29.9124 & 24.5395 \\
\hline MDNB & 3.7741 & 25.24254 & 20.96924 & -1.1592 & 36.62645 & 31.88075 & -0.3065 & 30.32022 & 24.21782 \\
\hline MDNB & 6.2805 & 22.49423 & 19.48843 & -2.8482 & 36.9256 & 32.3182 & 0.0485 & 30.82164 & 23.99344 \\
\hline DNBAL & 11.7026 & 18.27568 & 15.20258 & -0.0194 & 34.39411 & 31.05931 & 1.9851 & 29.69962 & 24.64432 \\
\hline DNBAL & 13.319 & 18.85226 & 16.77136 & -1.4203 & 34.16315 & 30.43865 & 0.8771 & 28.94532 & 24.01862 \\
\hline DNBAL & 12.5731 & 18.44348 & 16.26878 & -3.1831 & 36.42619 & 31.43469 & 2.0349 & 29.86628 & 23.45248 \\
\hline DNBAL & 12.4274 & 17.07362 & 15.32112 & 0.6491 & 34.21494 & 29.98544 & 1.1969 & 29.68498 & 24.42048 \\
\hline DNBAL & 14.1567 & 17.99314 & 15.44824 & 0.5212 & 34.34396 & 31.39726 & 0.6307 & 29.65595 & 23.57805 \\
\hline DNBAL & 11.9414 & 18.53855 & 14.79625 & 0.2926 & 33.56757 & 30.00977 & 2.0351 & 28.8075 & 22.9985 \\
\hline DNP & 18.7984 & 12.99834 & 11.46674 & -0.8214 & 33.55605 & 27.99635 & 0.8471 & 30.52332 & 22.60602 \\
\hline DNP & 19.1845 & 13.37664 & 11.06884 & 1.3485 & 32.41185 & 28.35935 & 1.1003 & 30.30386 & 21.31456 \\
\hline DNP & 18.7371 & 12.83263 & 11.68083 & 1.2719 & 32.80184 & 28.36194 & 2.4744 & 29.19475 & 21.28775 \\
\hline DNP & 16.4211 & 14.59833 & 11.64293 & 1.5263 & 32.20165 & 28.05195 & 2.1309 & 29.59065 & 21.89635 \\
\hline DNP & 20.0134 & 12.85979 & 10.88699 & 1.0731 & 33.38892 & 28.74392 & 2.1804 & 28.76144 & 21.47584 \\
\hline DNP & 18.8367 & 13.51204 & 10.82094 & 2.4232 & 32.52442 & 27.45732 & 1.3883 & 29.27772 & 22.54812 \\
\hline DNA & 4.2875 & 25.16351 & 19.12461 & 1.1819 & 32.84828 & 28.26708 & 1.5882 & 28.45614 & 23.19314 \\
\hline DNA & 3.7713 & 26.78677 & 20.35177 & 1.2453 & 32.81986 & 30.20966 & 1.1972 & 29.25292 & 23.95882 \\
\hline DNA & 3.9823 & 27.49389 & 21.20469 & -0.3862 & 32.68421 & 28.11151 & 1.0596 & 29.12783 & 23.08023 \\
\hline DNA & 4.8518 & 25.01984 & 19.90544 & 0.8634 & 32.80062 & 28.51752 & 3.8929 & 28.06091 & 22.96931 \\
\hline DNA & 3.6303 & 25.34405 & 20.08245 & -0.043 & 34.33051 & 28.59341 & 4.5537 & 27.53551 & 21.61741 \\
\hline DNA & 3.5686 & 25.86935 & 19.23835 & 1.0806 & 34.55706 & 29.94626 & 2.9221 & 27.73515 & 23.38085 \\
\hline DNSA & 18.0623 & 13.43552 & 7.69892 & 22.4268 & 14.66728 & 10.89208 & 19.5866 & 12.54395 & 9.20355 \\
\hline DNSA & 18.4954 & 11.38868 & 7.46048 & 23.5531 & 13.80918 & 10.96488 & 20.6362 & 12.14745 & 9.38385 \\
\hline DNSA & 17.9102 & 11.70256 & 6.82096 & 21.2656 & 14.75128 & 11.06748 & 19.4617 & 12.32735 & 8.32985 \\
\hline DNSA & 20.2363 & 11.77235 & 7.22965 & 22.0154 & 14.46649 & 10.46619 & 18.6499 & 12.79215 & 8.84545 \\
\hline DNSA & 19.406 & 12.93033 & 7.18833 & 22.3176 & 14.7296 & 11.5017 & 20.2774 & 11.69732 & 9.14872 \\
\hline
\end{tabular}




\begin{tabular}{|c|c|c|c|c|c|c|c|c|c|}
\hline DNSA & 18.5649 & 11.26081 & 6.31841 & 21.5119 & 14.73779 & 11.51889 & 18.4142 & 12.43105 & 8.27805 \\
\hline 4-NA & 7.8088 & 23.50113 & 15.86493 & 5.3633 & 30.01914 & 25.25634 & 6.9127 & 23.99307 & 19.32637 \\
\hline 4-NA & 10.9294 & 22.9467 & 17.1721 & 6.689 & 28.40706 & 24.76526 & 6.9703 & 25.76802 & 20.17552 \\
\hline 4-NA & 9.1077 & 23.06424 & 16.01384 & 4.2759 & 29.86921 & 25.77491 & 5.6238 & 25.42354 & 19.21774 \\
\hline 4-NA & 9.6991 & 22.58446 & 15.94446 & 4.585 & 30.38196 & 26.09466 & 8.0601 & 24.47183 & 20.05673 \\
\hline 4-NA & 8.9561 & 22.61967 & 17.11017 & 4.4777 & 31.42608 & 26.85668 & 7.0799 & 24.31394 & 20.05574 \\
\hline 4-NA & 10.7217 & 22.24393 & 16.92303 & 6.041 & 29.13017 & 25.43417 & 7.5686 & 24.37476 & 19.74166 \\
\hline $2 \mathrm{~A} 3 \mathrm{NP}$ & 6.2287 & 25.70097 & 19.11517 & -2.1584 & 36.88691 & 27.94221 & 1.8194 & 29.70649 & 21.60469 \\
\hline 2A3NP & 6.7521 & 25.94472 & 18.64882 & -1.8035 & 35.68738 & 28.18738 & 3.3925 & 29.1816 & 21.6013 \\
\hline 2A3NP & 4.3239 & 27.38258 & 17.45238 & -0.9522 & 34.48161 & 27.18521 & 1.6947 & 28.86716 & 21.60236 \\
\hline 2A3NP & 3.9154 & 26.85754 & 17.46484 & -1.2056 & 35.37672 & 26.76372 & 0.7061 & 29.97299 & 21.29989 \\
\hline 2A3NP & 4.2107 & 26.5622 & 17.5872 & -1.9223 & 36.30938 & 28.15528 & 1.6774 & 28.56497 & 21.18367 \\
\hline 2A3NP & 6.4845 & 25.62629 & 18.97069 & 0.1035 & 35.12493 & 28.36733 & 1.1886 & 30.26997 & 22.40807 \\
\hline PA & 24.7323 & 6.03586 & 4.13161 & 28.7844 & 7.90074 & 6.82984 & 25.1474 & 7.17044 & 6.13336 \\
\hline PA & 24.4741 & 7.14968 & 4.15792 & 29.9158 & 7.12933 & 6.08203 & 25.5412 & 7.39862 & 6.18455 \\
\hline PA & 24.4384 & 6.33039 & 4.0544 & 29.4994 & 7.4907 & 6.8147 & 24.7699 & 8.07223 & 6.67703 \\
\hline PA & 26.3507 & 6.5526 & 2.85596 & 29.5861 & 7.29871 & 6.28911 & 25.4317 & 7.53235 & 6.44255 \\
\hline PA & 25.3636 & 6.17048 & 4.26783 & 31.4287 & 6.44756 & 5.5807 & 24.4923 & 6.71716 & 5.75984 \\
\hline PA & 24.3357 & 6.57552 & 3.52484 & 30.1308 & 7.49828 & 7.14348 & 23.7355 & 7.8812 & 5.98526 \\
\hline CINP & 9.6195 & 23.94782 & 18.63202 & 1.3025 & 34.27096 & 30.20936 & 0.4338 & 29.61769 & 22.33629 \\
\hline CINP & 10.4904 & 22.8827 & 17.9457 & -1.6264 & 33.73353 & 28.70293 & 2.4023 & 29.82007 & 22.97617 \\
\hline CINP & 10.9022 & 24.09425 & 18.78305 & -0.6256 & 35.67264 & 28.58274 & 1.8767 & 28.78665 & 23.72415 \\
\hline CINP & 8.8765 & 24.68061 & 17.47321 & -0.9615 & 34.7304 & 29.5824 & 2.4464 & 28.9578 & 24.0457 \\
\hline CINP & 11.1287 & 22.81143 & 18.68253 & -0.7761 & 35.47401 & 29.62621 & 2.1413 & 28.55573 & 23.30623 \\
\hline CINP & 10.3203 & 24.68303 & 19.41963 & 0.2971 & 35.03322 & 29.59122 & 0.9534 & 30.09299 & 22.48049 \\
\hline 2A6NP & 9.5846 & 18.70406 & 13.24186 & 6.1069 & 28.4405 & 24.6301 & 8.1777 & 23.02624 & 18.98434 \\
\hline 2A6NP & 10.8021 & 18.08588 & 11.74108 & 6.8103 & 27.11597 & 22.63907 & 8.716 & 22.25492 & 18.78262 \\
\hline 2A6NP & 9.8817 & 18.79359 & 11.92299 & 7.3382 & 25.93697 & 20.63457 & 8.3617 & 22.50049 & 18.80079 \\
\hline 2A6NP & 10.5495 & 19.79089 & 13.47069 & 6.9608 & 27.52538 & 23.34438 & 7.8729 & 22.93424 & 17.86294 \\
\hline 2A6NP & 9.8639 & 20.33277 & 14.42427 & 6.6577 & 27.67426 & 22.51526 & 7.4987 & 23.20598 & 19.09528 \\
\hline 2A6NP & 9.8067 & 19.36306 & 11.73976 & 8.6603 & 26.19785 & 22.59475 & 8.661 & 22.55425 & 18.93235 \\
\hline DNBA & 15.9057 & 13.62227 & 11.07747 & -1.9957 & 35.91995 & 29.35925 & 1.0078 & 30.34405 & 23.69645 \\
\hline DNBA & 15.9805 & 14.31623 & 10.63923 & -1.557 & 34.87015 & 30.30545 & 1.4415 & 30.1203 & 24.0236 \\
\hline
\end{tabular}




\begin{tabular}{|c|c|c|c|c|c|c|c|c|c|}
\hline DNBA & 15.9116 & 14.34433 & 10.34273 & -3.5349 & 35.32603 & 29.93593 & 0.5644 & 29.88889 & 24.01659 \\
\hline DNBA & 14.4334 & 15.46178 & 10.36038 & -1.3957 & 35.23857 & 30.56567 & 0.6663 & 29.52322 & 24.56132 \\
\hline DNBA & 15.5229 & 13.73976 & 10.25096 & -1.1372 & 35.13064 & 30.20354 & -0.4963 & 30.03079 & 24.38499 \\
\hline DNBA & 16.623 & 14.30822 & 10.13652 & -1.5347 & 35.33801 & 29.50691 & 0.6634 & 29.27895 & 22.67385 \\
\hline $4 \mathrm{NP}$ & 11.3508 & 18.67117 & 14.30317 & 11.1206 & 23.62555 & 20.68425 & 10.5354 & 20.05848 & 17.00818 \\
\hline $4 \mathrm{NP}$ & 11.338 & 19.29929 & 15.59459 & 11.1378 & 23.30813 & 20.41593 & 11.2427 & 20.67924 & 16.53624 \\
\hline $4 \mathrm{NP}$ & 12.0518 & 19.67764 & 15.74944 & 12.1656 & 22.86055 & 20.13275 & 11.5617 & 19.3373 & 16.7287 \\
\hline $4 \mathrm{NP}$ & 13.187 & 18.62965 & 14.70495 & 10.1905 & 23.97719 & 19.59859 & 11.3232 & 20.05679 & 17.50809 \\
\hline $4 \mathrm{NP}$ & 10.3308 & 20.15787 & 16.15897 & 11.9442 & 23.64826 & 20.76166 & 10.5326 & 21.03689 & 17.34329 \\
\hline $4 \mathrm{NP}$ & 12.4707 & 20.1494 & 15.9153 & 10.272 & 24.70245 & 20.05205 & 7.8668 & 22.29216 & 19.30826 \\
\hline
\end{tabular}

Table S6: Dataset for unknown samples analysis by using native quantum dot array.

\begin{tabular}{|c|c|c|c|c|c|c|c|c|c|}
\hline $\begin{array}{l}\text { SI } \\
\text { No }\end{array}$ & AuNC & $\begin{array}{l}\text { AuNC } \\
+Q A\end{array}$ & $\begin{array}{c}\text { AuNC } \\
+\mathrm{QA}+\mathrm{MA}\end{array}$ & MQD & $\begin{array}{l}\text { MQD } \\
+Q A\end{array}$ & $\begin{array}{c}\text { MQD } \\
+\mathrm{QA}+\mathrm{MA}\end{array}$ & WQD & $\begin{array}{l}\text { WQD } \\
+Q A\end{array}$ & $\begin{array}{c}\text { WQD } \\
+\mathrm{QA}+\mathrm{MA}\end{array}$ \\
\hline 1 & -0.3815 & 27.72393 & 22.41623 & -0.8064 & 35.8983 & 21.9778 & 0.5217 & $\begin{array}{c}40.263 \\
6 \\
\end{array}$ & 34.0321 \\
\hline 2 & 0.4767 & 26.79409 & 20.44539 & 0.5644 & 33.8275 & 22.6698 & 1.4088 & $\begin{array}{c}39.171 \\
7\end{array}$ & 34.487 \\
\hline 3 & -1.7314 & 28.3903 & 22.2562 & 0.6716 & 33.413 & 21.2828 & 0.4791 & $\begin{array}{c}39.207 \\
8\end{array}$ & 34.0513 \\
\hline 4 & 1.2755 & 27.29565 & 21.98025 & 2.0302 & 34.8936 & 21.0197 & 0.848 & $\begin{array}{c}38.531 \\
3\end{array}$ & 34.9059 \\
\hline 5 & -0.512 & 28.37257 & 20.47607 & -1.3988 & 33.2041 & 20.2819 & 0.3141 & $\begin{array}{c}40.054 \\
7 \\
\end{array}$ & 33.4232 \\
\hline 6 & 0.5603 & 28.11964 & 22.66004 & 0.1654 & 34.5428 & 21.6657 & 0.563 & $\begin{array}{c}39.265 \\
3 \\
\end{array}$ & 33.0085 \\
\hline 7 & 0.8294 & 28.68932 & 21.20902 & 9.7084 & 28.7012 & 13.923 & 6.6085 & $\begin{array}{c}30.641 \\
8\end{array}$ & 27.194 \\
\hline 8 & 1.2244 & 28.72402 & 20.93272 & 9.5664 & 27.7882 & 14.7292 & 5.7546 & $\begin{array}{c}28.593 \\
6\end{array}$ & 26.4734 \\
\hline 9 & -0.4013 & 30.35617 & 20.20727 & 9.4116 & 26.8027 & 13.5622 & 7.5333 & $\begin{array}{c}30.492 \\
9 \\
\end{array}$ & 26.1194 \\
\hline 10 & 3.348 & 26.99514 & 21.05024 & 10.1071 & 27.6138 & 15.362 & 6.5386 & $\begin{array}{c}30.392 \\
4 \\
\end{array}$ & 26.6204 \\
\hline 11 & 0.0742 & 28.30759 & 20.56709 & 9.7609 & 26.4767 & 14.9272 & 6.7427 & $\begin{array}{c}30.994 \\
3\end{array}$ & 26.475 \\
\hline 12 & 0.6742 & 29.6712 & 19.8583 & 8.7605 & 25.1016 & 14.1509 & 5.1565 & $\begin{array}{c}28.177 \\
9 \\
\end{array}$ & 25 \\
\hline 13 & 0.7784 & 28.88168 & 21.37788 & 2.4579 & 31.5584 & 20.6866 & 2.3847 & $\begin{array}{c}36.039 \\
9\end{array}$ & 28.8767 \\
\hline 14 & 0.2398 & 29.14543 & 21.72913 & 1.1689 & 31.9906 & 19.2975 & 2.6818 & $\begin{array}{c}35.918 \\
5 \\
\end{array}$ & 30.2168 \\
\hline 15 & 2.0845 & 29.41254 & 21.88694 & 2.6003 & 31.4636 & 20.2746 & 2.8196 & $\begin{array}{c}35.705 \\
6\end{array}$ & 28.3992 \\
\hline 16 & 0.3906 & 29.52997 & 21.45947 & 2.1555 & 29.4932 & 20.3288 & 0.079 & $\begin{array}{c}36.652 \\
2 \\
\end{array}$ & 27.7784 \\
\hline 17 & -0.6037 & 29.59031 & 20.75661 & 2.0515 & 32.4038 & 20.5214 & 1.4576 & $\begin{array}{c}37.136 \\
2 \\
\end{array}$ & 29.9109 \\
\hline 18 & 0.5499 & 29.92175 & 21.26245 & 2.1309 & 32.5961 & 19.7644 & 2.2671 & $\begin{array}{c}34.994 \\
8 \\
\end{array}$ & 29.8397 \\
\hline 19 & 5.4945 & 25.76399 & 18.73079 & 1.2726 & 31.4064 & 22.1389 & 1.9996 & $\begin{array}{c}37.965 \\
3\end{array}$ & 32.4761 \\
\hline
\end{tabular}




\begin{tabular}{|c|c|c|c|c|c|c|c|c|c|}
\hline 20 & 7.7069 & 24.8502 & 18.8658 & 1.4589 & 33.8945 & 22.1006 & 1.0142 & $\begin{array}{c}35.622 \\
8\end{array}$ & 30.4616 \\
\hline 21 & 4.7717 & 27.08943 & 19.54243 & -4.3827 & 32.5939 & 23.8949 & 2.3433 & $\begin{array}{c}38.550 \\
6\end{array}$ & 30.283 \\
\hline 22 & 6.8477 & 25.75148 & 18.24578 & 2.4417 & 31.3347 & 23.3569 & 1.5475 & $\begin{array}{c}37.935 \\
5\end{array}$ & 31.2046 \\
\hline 23 & 7.9566 & 25.96908 & 19.40298 & 1.7074 & 31.4916 & 21.1948 & -0.1336 & $\begin{array}{c}35.915 \\
5 \\
\end{array}$ & 31.4784 \\
\hline 24 & 6.8625 & 25.97909 & 19.37539 & 0.7957 & 30.9319 & 20.1994 & 1.362 & $\begin{array}{c}37.056 \\
5\end{array}$ & 31.6477 \\
\hline 25 & 15.7775 & 17.46783 & 14.50333 & 16.0001 & 17.8797 & 6.0861 & 18.18 & $\begin{array}{c}22.045 \\
4 \\
\end{array}$ & 14.73794 \\
\hline 26 & 12.4834 & 19.8759 & 14.4582 & 17.668 & 17.7151 & 6.2191 & 18.2738 & 20.597 & 17.07026 \\
\hline 27 & 13.9051 & 17.66681 & 12.98951 & 19.8126 & 18.1291 & 6.9763 & 19.0031 & $\begin{array}{c}21.848 \\
6 \\
\end{array}$ & 14.19089 \\
\hline 28 & 13.4688 & 16.97821 & 12.34721 & 18.4423 & 17.1371 & 7.2647 & 17.9037 & $\begin{array}{c}22.865 \\
9\end{array}$ & 15.04739 \\
\hline 29 & 13.0322 & 18.99118 & 12.62548 & 16.8759 & 18.5887 & 5.3096 & 19.0517 & $\begin{array}{c}21.641 \\
6\end{array}$ & 14.19064 \\
\hline 30 & 15.0807 & 17.39745 & 12.61025 & 18.2851 & 18.96 & 6.1709 & 17.8304 & $\begin{array}{c}22.767 \\
4\end{array}$ & 15.00976 \\
\hline 31 & 5.3289 & 25.31772 & 19.12882 & 5.4287 & $\begin{array}{c}28.7292 \\
7 \\
\end{array}$ & 13.4757 & 6.5613 & $\begin{array}{c}32.129 \\
8 \\
\end{array}$ & 24.3756 \\
\hline 32 & 4.9963 & 25.34777 & 18.67717 & 5.4318 & $\begin{array}{c}28.2760 \\
7 \\
\end{array}$ & 13.2595 & 6.4095 & $\begin{array}{c}31.818 \\
2\end{array}$ & 25.1806 \\
\hline 33 & 5.464 & 24.2531 & 17.092 & 6.4269 & $\begin{array}{c}31.2084 \\
4 \\
\end{array}$ & 13.9805 & 7.7859 & $\begin{array}{c}30.146 \\
6 \\
\end{array}$ & 26.1378 \\
\hline 34 & 5.0415 & 24.88965 & 18.12905 & 4.1487 & $\begin{array}{c}28.1090 \\
2 \\
\end{array}$ & 15.6594 & 6.4379 & $\begin{array}{c}32.491 \\
6 \\
\end{array}$ & 26.7072 \\
\hline 35 & 6.1519 & 26.28297 & 18.41827 & 4.0577 & $\begin{array}{c}27.5201 \\
7\end{array}$ & 14.1237 & 7.4778 & 32.773 & 26.6047 \\
\hline 36 & 5.4716 & 25.24598 & 18.55348 & 3.9694 & $\begin{array}{c}27.8512 \\
3 \\
\end{array}$ & 14.644 & 7.9693 & 31.487 & 25.9285 \\
\hline 37 & 5.2768 & 24.03855 & 18.53105 & -0.0031 & 33.1688 & 18.5832 & -0.0946 & $\begin{array}{c}36.345 \\
4\end{array}$ & 28.5899 \\
\hline 38 & 3.9494 & 25.1452 & 16.7201 & 0.6937 & 33.124 & 17.04117 & 3.0323 & $\begin{array}{c}35.703 \\
7 \\
\end{array}$ & 27.2382 \\
\hline 39 & 6.3867 & 22.80475 & 17.60955 & 1.5813 & 32.1607 & 17.43098 & 2.6179 & $\begin{array}{c}36.922 \\
4\end{array}$ & 30.1222 \\
\hline 40 & 5.0266 & 23.81452 & 17.37272 & 1.209 & 34.0317 & 17.62342 & 2.2014 & $\begin{array}{c}33.527 \\
3 \\
\end{array}$ & 28.1873 \\
\hline 41 & 5.4125 & 23.97822 & 18.30032 & 0.8092 & 32.1914 & 17.15045 & 1.0886 & $\begin{array}{c}37.957 \\
2 \\
\end{array}$ & 28.5146 \\
\hline 42 & 5.1217 & 22.84408 & 17.75898 & 1.8119 & 31.8348 & 17.00634 & 0.6296 & $\begin{array}{c}35.338 \\
4 \\
\end{array}$ & 29.8185 \\
\hline 43 & 21.5041 & 8.95633 & 8.42823 & 24.2648 & 11.8018 & 5.9934 & $\begin{array}{c}25.6024 \\
4 \\
\end{array}$ & $\begin{array}{c}10.736 \\
6 \\
\end{array}$ & 10.7397 \\
\hline 44 & 21.353 & 8.61329 & 8.09799 & 22.6108 & 11.5873 & 5.1725 & $\begin{array}{c}25.0445 \\
6 \\
\end{array}$ & $\begin{array}{c}11.334 \\
8 \\
\end{array}$ & 12.7337 \\
\hline 45 & 23.6278 & 8.23848 & 7.39618 & 21.8566 & 10.9321 & 5.2382 & $\begin{array}{c}26.1541 \\
5 \\
\end{array}$ & $\begin{array}{c}10.059 \\
2 \\
\end{array}$ & 11.9348 \\
\hline 46 & 21.163 & 8.88205 & 7.68435 & 23.9859 & 13.1185 & 4.7476 & $\begin{array}{c}25.8093 \\
6 \\
\end{array}$ & $\begin{array}{c}11.690 \\
9 \\
\end{array}$ & 12.5766 \\
\hline 47 & 21.1206 & 9.08481 & 8.15121 & 24.4266 & 11.8882 & 4.8403 & 25.6392 & $\begin{array}{c}11.659 \\
7\end{array}$ & 10.2198 \\
\hline 48 & 22.1776 & 8.78105 & 8.23855 & 25.5139 & 11.4838 & 5.7814 & 24.5728 & $\begin{array}{c}12.419 \\
1 \\
\end{array}$ & 11.5426 \\
\hline 49 & 2.2598 & 26.23161 & 20.04541 & 7.9761 & 23.2892 & 10.8017 & 9.6458 & $\begin{array}{c}26.873 \\
3 \\
\end{array}$ & 23.59052 \\
\hline 50 & 1.2751 & 27.32682 & 19.32722 & 6.8269 & 23.0741 & 11.6907 & 9.5685 & $\begin{array}{c}26.695 \\
3\end{array}$ & 23.50672 \\
\hline 51 & 1.3193 & 27.82499 & 20.77139 & 8.2302 & 23.4761 & 11.494 & 9.8968 & $\begin{array}{c}26.989 \\
1\end{array}$ & 22.2796 \\
\hline
\end{tabular}




\begin{tabular}{|l|l|l|l|l|l|l|l|l|l|}
$\mathbf{5 2}$ & 3.7416 & 27.81408 & 21.44248 & 9.1768 & 23.4654 & 11.018 & 12.345 & $\begin{array}{c}27.770 \\
1\end{array}$ & 23.1264 \\
\hline $\mathbf{5 3}$ & 3.2167 & 27.01688 & 20.76518 & 9.4285 & 24.4996 & 11.0097 & 10.2517 & $\begin{array}{c}27.649 \\
7\end{array}$ & 23.67616 \\
\hline $\mathbf{5 4}$ & 2.431 & 26.19433 & 18.11553 & 7.7941 & 21.5429 & 11.5172 & 11.391 & $\begin{array}{c}27.897 \\
2\end{array}$ & 22.60513 \\
\hline
\end{tabular}

Table S7: Verification of observed sample with expected analyte by using native quantum dot array in different channels.

\begin{tabular}{|c|c|c|c|c|}
\hline SI No & Expected & Observed ( 3 Channel) & Observed (6 Channel) & Observed ( 9 Channel) \\
\hline 1 & $2 \mathrm{NP}$ & $2 \mathrm{NP}$ & $2 \mathrm{NP}$ & $2 \mathrm{NP}$ \\
\hline 2 & $2 N P$ & $2 \mathrm{NP}$ & $2 N P$ & $2 \mathrm{NP}$ \\
\hline 3 & $2 \mathrm{NP}$ & $2 \mathrm{NP}$ & $2 \mathrm{NP}$ & $2 \mathrm{NP}$ \\
\hline 4 & $2 N P$ & DNP & $2 \mathrm{NP}$ & $2 \mathrm{NP}$ \\
\hline 5 & $2 N P$ & $2 \mathrm{NP}$ & $2 \mathrm{NP}$ & $2 \mathrm{NP}$ \\
\hline 6 & $2 \mathrm{NP}$ & $2 \mathrm{NP}$ & $2 \mathrm{NP}$ & $2 \mathrm{NP}$ \\
\hline 7 & $3 M 4 N P$ & $3 M 4 N P$ & $3 M 4 N P$ & $3 M 4 N P$ \\
\hline 8 & $3 M 4 N P$ & $3 M 4 N P$ & $3 M 4 N P$ & $3 M 4 N P$ \\
\hline 9 & $3 M 4 N P$ & $3 M 4 N P$ & $3 M 4 N P$ & $3 M 4 N P$ \\
\hline 10 & 3M4NP & $3 M 4 N P$ & $3 M 4 N P$ & $3 M 4 N P$ \\
\hline 11 & $3 M 4 N P$ & $3 M 4 N P$ & $3 M 4 N P$ & $3 M 4 N P$ \\
\hline 12 & $3 M 4 N P$ & $3 M 4 N P$ & 4NP & 4NP \\
\hline 13 & DNP & DNP & DNP & DNP \\
\hline 14 & DNP & DNP & DNP & DNP \\
\hline 15 & DNP & 2A3NP & DNP & DNP \\
\hline 16 & DNP & $2 \mathrm{NP}$ & DNP & DNP \\
\hline 17 & DNP & DNP & DNP & DNP \\
\hline 18 & DNP & DNP & DNP & DNP \\
\hline 19 & NBA & NOT PREDICTABLE & NOT PREDICTABLE & NBA \\
\hline 20 & NBA & NOT PREDICTABLE & NOT PREDICTABLE & NBA \\
\hline 21 & NBA & NOT PREDICTABLE & NOT PREDICTABLE & NBA \\
\hline 22 & NBA & NOT PREDICTABLE & NOT PREDICTABLE & NBA \\
\hline 23 & NBA & NOT PREDICTABLE & NOT PREDICTABLE & NBA \\
\hline 24 & NBA & NOT PREDICTABLE & NOT PREDICTABLE & NBA \\
\hline 25 & DNSA & DNSA & DNSA & DNSA \\
\hline 26 & DNSA & DNSA & DNSA & DNSA \\
\hline 27 & DNSA & DNSA & DNSA & DNSA \\
\hline 28 & DNSA & DNSA & DNSA & DNSA \\
\hline 29 & DNSA & DNSA & DNSA & DNSA \\
\hline 30 & DNSA & DNSA & DNSA & DNSA \\
\hline 31 & 4-NA & 4-NA & 4-NA & 4-NA \\
\hline 32 & 4-NA & 4-NA & 4-NA & 4-NA \\
\hline
\end{tabular}




\begin{tabular}{|c|c|c|c|c|}
\hline 33 & 4-NA & 4-NA & NBA & 2A3NP \\
\hline 34 & 4-NA & 4-NA & 4-NA & 4-NA \\
\hline 35 & 4-NA & 4-NA & 4-NA & 4-NA \\
\hline 36 & 4-NA & 4-NA & 4-NA & 4-NA \\
\hline 37 & 2A3NP & NOT PREDICTABLE & NOT PREDICTABLE & 2A3NP \\
\hline 38 & 2A3NP & NOT PREDICTABLE & NOT PREDICTABLE & 2A3NP \\
\hline 39 & 2A3NP & NOT PREDICTABLE & NOT PREDICTABLE & 2A3NP \\
\hline 40 & 2A3NP & NOT PREDICTABLE & NOT PREDICTABLE & 2A3NP \\
\hline 41 & 2A3NP & NOT PREDICTABLE & NOT PREDICTABLE & 2A3NP \\
\hline 42 & 2A3NP & NOT PREDICTABLE & NOT PREDICTABLE & 2A3NP \\
\hline 43 & $\mathrm{PA}$ & PA & PA & PA \\
\hline 44 & PA & PA & PA & PA \\
\hline 45 & PA & PA & PA & PA \\
\hline 46 & PA & PA & PA & PA \\
\hline 47 & PA & PA & PA & PA \\
\hline 48 & PA & PA & PA & PA \\
\hline 49 & $4 \mathrm{NP}$ & $3 M 4 N P$ & $4 \mathrm{NP}$ & $4 \mathrm{NP}$ \\
\hline 50 & $4 \mathrm{NP}$ & $3 M 4 N P$ & $4 \mathrm{NP}$ & $4 \mathrm{NP}$ \\
\hline 51 & $4 \mathrm{NP}$ & $3 M 4 N P$ & $4 \mathrm{NP}$ & $4 \mathrm{NP}$ \\
\hline 52 & $4 \mathrm{NP}$ & $4 \mathrm{NP}$ & $4 \mathrm{NP}$ & $4 \mathrm{NP}$ \\
\hline 53 & $4 \mathrm{NP}$ & $4 \mathrm{NP}$ & $4 \mathrm{NP}$ & $4 \mathrm{NP}$ \\
\hline 54 & $4 \mathrm{NP}$ & $4 \mathrm{NP}$ & $4 \mathrm{NP}$ & $4 \mathrm{NP}$ \\
\hline
\end{tabular}

Table S8: Dataset for unknown samples analysis by using thiol-functionalized quantum dot array.

\begin{tabular}{|c|c|c|c|c|c|c|c|c|c|}
\hline SI No & BA AuNC & $\begin{array}{c}\text { BA AuNC } \\
+ \text { QA }\end{array}$ & $\begin{array}{r}\text { BA AuNC } \\
+\mathrm{QA}+\mathrm{MA} \\
\end{array}$ & BA MQD & $\begin{array}{c}\text { BA MQD } \\
+ \text { QA }\end{array}$ & $\begin{array}{c}\text { BA MQD } \\
+\mathrm{QA}+\mathrm{MA} \\
\end{array}$ & BA WQD & $\begin{array}{c}\text { BA WQD } \\
+ \text { QA }\end{array}$ & $\begin{array}{c}\text { BA WQD } \\
+\mathrm{QA}+\mathrm{MA} \\
\end{array}$ \\
\hline 1 & 0.7665 & 28.6565 & 21.65 & -0.1376 & 35.158 & 30.0703 & 1.7318 & 30.397 & 23.8631 \\
\hline 2 & 0.6724 & 28.4465 & 21.1863 & 0.5092 & 29.3936 & 30.2822 & 1.84503 & 30.6964 & 23.0576 \\
\hline 3 & 0.9911 & 27.1758 & 21.2237 & -0.2426 & 34.823 & 31.1635 & 2.35422 & 29.9945 & 22.4255 \\
\hline 4 & -0.5541 & 27.9652 & 21.4131 & 0.5864 & 35.264 & 31.2573 & 1.67865 & 30.4688 & 23.5675 \\
\hline 5 & -0.438 & 25.9711 & 21.6525 & -0.0864 & 34.8264 & 29.5636 & 1.89442 & 29.6401 & 23.9737 \\
\hline 6 & 0.5621 & 27.265 & 20.2608 & -0.7908 & 34.5975 & 30.4153 & 1.19374 & 29.6503 & 23.2611 \\
\hline 7 & 5.63405 & 19.0972 & 20.78703 & -0.362 & 36.3915 & 30.97812 & 0.3212 & 29.89207 & 25.6699 \\
\hline 8 & 5.37197 & 19.0126 & 20.44551 & -1.0719 & 35.16409 & 32.1966 & 0.2517 & 29.92196 & 24.7393 \\
\hline 9 & 5.28723 & 19.6598 & 20.76094 & -1.4245 & 34.90368 & 32.54769 & -0.6109 & 29.62634 & 26.3102 \\
\hline 10 & 6.15874 & 20.55503 & 20.64568 & -1.0022 & 35.73513 & 37.89658 & 0.5789 & 29.51843 & 24.985 \\
\hline 11 & 5.30917 & 20.5746 & 21.0317 & -0.4911 & 36.44025 & 31.82866 & -1.0293 & 29.25225 & 27.22172 \\
\hline 12 & 5.95787 & 18.9422 & 20.24778 & -1.0931 & 36.04815 & 32.32122 & -0.1582 & 30.14454 & 23.6986 \\
\hline 13 & 12.79673 & 18.67759 & 15.2524 & 2.51942 & 34.7212 & 30.43427 & 0.88884 & 28.60515 & 24.38896 \\
\hline 14 & 12.62345 & 18.45971 & 15.8359 & 1.5983 & 34.0263 & 30.60667 & 1.57954 & 28.61864 & 24.58284 \\
\hline
\end{tabular}




\begin{tabular}{|c|c|c|c|c|c|c|c|c|c|}
\hline 15 & 12.79159 & 18.62123 & 15.3796 & 0.5021 & 34.9594 & 31.11477 & 0.0682 & 28.59764 & 23.53149 \\
\hline 16 & 12.76855 & 18.23122 & 14.7223 & 1.0109 & 36.0799 & 30.41352 & 0.4987 & 30.201 & 24.60406 \\
\hline 17 & 12.83597 & 18.48199 & 14.8297 & -0.1315 & 34.3957 & 31.18937 & 1.33807 & 28.10867 & 24.66833 \\
\hline 18 & 12.7369 & 18.39025 & 15.5967 & 0.6043 & 34.5515 & 31.13547 & 1.32291 & 28.43561 & 24.30635 \\
\hline 19 & 18.1118 & 12.70609 & 10.66155 & 2.23129 & 34.41546 & 28.6976 & 2.4189 & 30.60838 & 21.81932 \\
\hline 20 & 16.4718 & 12.92387 & 10.51391 & 2.33014 & 33.55899 & 28.5468 & 2.00783 & 31.32803 & 22.5994 \\
\hline 21 & 18.3967 & 13.10211 & 10.12816 & 2.11048 & 33.96859 & 29.2395 & 2.15727 & 30.77903 & 22.40626 \\
\hline 22 & 18.3025 & 12.12265 & 10.56277 & 1.27488 & 33.14046 & 28.3624 & 2.4158 & 30.74598 & 21.63415 \\
\hline 23 & 17.5866 & 9.3599 & 10.39335 & 1.55974 & 33.31162 & 28.3737 & 2.294 & 31.42826 & 22.66983 \\
\hline 24 & 18.1593 & 13.96785 & 10.59352 & 1.98298 & 33.54121 & 27.5949 & 1.1442 & 30.73255 & 21.47945 \\
\hline 25 & -1.2183 & 24.6775 & 20.39619 & 0.25767 & 33.16859 & 28.70572 & 1.72881 & 28.00393 & 22.70304 \\
\hline 26 & 3.3892 & 25.6232 & 20.52591 & 0.23828 & 33.0827 & 28.19302 & 2.27042 & 28.53201 & 22.65408 \\
\hline 27 & 3.3183 & 24.7717 & 20.14134 & 0.71873 & 32.5316 & 28.64899 & -2.7361 & 28.25691 & 22.58976 \\
\hline 28 & 3.0365 & 26.0499 & 20.22356 & 0.19252 & 33.25422 & 28.38697 & 2.29585 & 28.26853 & 22.81687 \\
\hline 29 & 5.0337 & 25.782 & 20.50481 & 0.45644 & 33.19611 & 28.34954 & 2.49058 & 27.8843 & 22.79912 \\
\hline 30 & 3.1177 & 24.6319 & 20.39037 & -1.22428 & 33.57144 & 28.68124 & 2.23907 & 27.8655 & 22.01803 \\
\hline 31 & 18.34349 & 11.17749 & 8.2228 & 21.24016 & 13.13568 & 10.83465 & 20.1095 & 13.10126 & 7.302671 \\
\hline 32 & 18.15756 & 11.22138 & 7.55687 & 21.61417 & 19.24053 & 9.97961 & 20.40709 & 12.93149 & 7.72787 \\
\hline 33 & 18.27115 & 13.69848 & 8.66553 & 21.38992 & 13.52622 & 10.25361 & 21.14588 & 13.34407 & 8.019295 \\
\hline 34 & 18.58824 & 11.56647 & 7.34777 & 22.48043 & 14.70723 & 10.66544 & 20.62971 & 12.63613 & 9.045162 \\
\hline 35 & 18.21986 & 11.99185 & 4.0072 & 21.1655 & 14.12696 & 9.68029 & 22.39501 & 13.29842 & 7.704686 \\
\hline 36 & 17.94763 & 12.34928 & 8.78063 & 21.44989 & 13.41295 & 10.22755 & 20.79402 & 13.4128 & 7.248924 \\
\hline 37 & 9.93205 & 23.81838 & 15.9335 & 5.01061 & 31.48116 & 25.8106 & 6.46539 & 21.38461 & 18.85714 \\
\hline 38 & 10.48947 & 21.10927 & 15.99596 & 5.58475 & 31.60763 & 25.7246 & 7.90569 & 20.88984 & 19.25811 \\
\hline 39 & 10.69889 & 24.04606 & 15.74984 & 6.3251 & 31.61644 & 26.314 & 6.28107 & 23.26748 & 19.702 \\
\hline 40 & 10.38832 & 23.99163 & 15.93567 & 5.04347 & 31.73407 & 26.5482 & 7.44106 & 21.85533 & 19.31759 \\
\hline 41 & 9.17706 & 23.64329 & 15.78797 & 6.90375 & 31.58462 & 25.2538 & 6.3124 & 20.94336 & 18.56735 \\
\hline 42 & 10.7995 & 23.50911 & 15.42065 & 6.32192 & 31.1613 & 26.3032 & 6.80242 & 20.9898 & 19.13106 \\
\hline 43 & 6.07446 & 25.83595 & 18.20501 & -0.8453 & 34.557 & 28.4207 & 1.61555 & 30.32983 & 21.16722 \\
\hline 44 & 6.07571 & 25.3193 & 18.49369 & -0.7568 & 34.98692 & 28.19733 & 2.0413 & 30.42581 & 23.00363 \\
\hline 45 & 6.27881 & 25.8357 & 18.08248 & -1.1855 & 35.70145 & 27.6325 & 1.49594 & 29.96016 & 20.88525 \\
\hline 46 & 6.37101 & 25.1379 & 17.94127 & -0.7313 & 34.2756 & 26.5133 & 0.8777 & 29.86998 & 22.87147 \\
\hline 47 & 6.42879 & 25.92818 & 17.09159 & -1.2087 & 35.86356 & 28.4206 & 1.66212 & 28.85531 & 21.02935 \\
\hline 48 & 6.14604 & 26.20711 & 18.17193 & -0.8547 & 34.87126 & 27.5252 & 1.1964 & 29.24738 & 21.8078 \\
\hline 49 & 23.75562 & 4.0324 & 2.68082 & 30.71837 & 5.67465 & 5.31557 & 25.60967 & 5.45373 & 7.00452 \\
\hline 50 & 23.74709 & 5.84916 & 3.78181 & 30.20084 & 5.86832 & 5.5206 & 25.26804 & 5.05087 & 5.48268 \\
\hline 51 & 23.79831 & 5.73436 & 1.75585 & 31.25666 & 4.75213 & 6.19015 & 24.34337 & 5.27884 & 5.68645 \\
\hline 52 & 23.97523 & 5.77574 & 2.55545 & 29.4522 & 5.14753 & 5.7111 & 26.0673 & 5.90732 & 5.26209 \\
\hline 53 & 24.37167 & 5.23439 & 3.28898 & 30.15824 & 5.20666 & 6.178 & 24.77206 & 6.05534 & 6.43643 \\
\hline 54 & 23.9191 & 6.22166 & 3.37631 & 30.26929 & 5.07547 & 5.67498 & 25.28391 & 4.57778 & 6.82824 \\
\hline 55 & 10.10295 & 23.54316 & 20.78866 & -0.9309 & 35.8497 & 30.98179 & 1.5484 & 29.1547 & 21.9678 \\
\hline
\end{tabular}




\begin{tabular}{|c|c|c|c|c|c|c|c|c|c|}
\hline 56 & 10.59003 & 24.46343 & 22.79808 & -1.1455 & 35.6333 & 31.54317 & 1.64698 & 31.8669 & 22.5903 \\
\hline 57 & 10.23757 & 23.91228 & 23.05195 & -1.5571 & 36.08253 & 31.09653 & 3.40752 & 29.5434 & 22.4395 \\
\hline 58 & 9.984 & 23.94001 & 23.22593 & -0.90194 & 35.299 & 29.45335 & 1.47588 & 28.9068 & 22.4298 \\
\hline 59 & 10.05261 & 23.52352 & 22.62857 & -0.48779 & 35.0883 & 31.3634 & 6.162 & 29.4425 & 22.8788 \\
\hline 60 & 10.93169 & 23.69944 & 22.99079 & -1.7332 & 35.3733 & 30.26304 & -6.6094 & 29.8411 & 22.8432 \\
\hline 61 & 9.43028 & 18.99665 & 12.3621 & 6.14134 & 28.0845 & 24.00319 & 9.26058 & 23.53285 & 20.25959 \\
\hline 62 & 9.602005 & 18.53052 & 10.75608 & 5.70682 & 29.72538 & 24.53616 & 9.31046 & 23.06109 & $19.6620 €$ \\
\hline 63 & 9.58241 & 18.16179 & 11.61362 & 6.79619 & 28.7235 & 23.84389 & 7.32246 & 22.94661 & 20.04099 \\
\hline 64 & 9.324855 & 18.45341 & 10.92459 & 6.24644 & 28.4668 & 24.1832 & 8.65018 & 23.96373 & 18.9523 \\
\hline 65 & 9.477555 & 19.6271 & 12.68084 & 6.31889 & 28.0997 & 24.47033 & 9.33005 & 23.87145 & 20.03667 \\
\hline 66 & 9.563085 & 19.63055 & 11.21749 & 6.62382 & 27.6578 & 25.08507 & 8.82156 & 24.08381 & 19.68618 \\
\hline 67 & 15.76421 & 13.49166 & 10.64923 & -2.0815 & 35.77997 & 30.08311 & -0.87375 & 30.71409 & 24.811 \\
\hline 68 & 16.03339 & 13.53897 & 10.71278 & -1.42815 & 37.15152 & 30.22467 & 0.3296 & 30.66645 & 25.9122 \\
\hline 69 & 15.71201 & 14.08242 & 9.20688 & -1.7118 & 35.65088 & 29.82314 & 0.12546 & 30.49283 & 25.1559 \\
\hline 70 & 15.9163 & 13.91722 & 10.89279 & -2.1362 & 34.929 & 30.52162 & -0.52313 & 30.69561 & 24.1027 \\
\hline 71 & 15.56004 & 13.71412 & 10.37497 & -1.92022 & 35.81719 & 29.58503 & -0.30076 & 31.34523 & 24.9071 \\
\hline 72 & 15.75282 & 13.57406 & 11.86441 & -2.17276 & 35.61965 & 30.33246 & -0.64379 & 30.70221 & 24.075 \\
\hline 73 & 11.7752 & 19.62982 & 12.98296 & 11.19972 & 23.48046 & 19.68884 & 10.53469 & 21.08146 & 16.89244 \\
\hline 74 & 12.32215 & 21.19798 & 14.82096 & 11.58985 & 23.57149 & 20.01969 & 10.24303 & 19.80257 & 16.43093 \\
\hline 75 & 8.1438 & 19.65647 & 13.88965 & 12.16879 & 23.28549 & 20.34322 & 10.76742 & 20.58886 & 16.45307 \\
\hline 76 & 12.1441 & 19.55991 & 13.19269 & 10.80899 & 23.2708 & 19.84325 & 10.95278 & 20.99246 & 15.30615 \\
\hline 77 & 11.6061 & 18.5144 & 13.82044 & 10.92515 & 23.45178 & 20.02328 & 10.90658 & 19.92031 & 15.50218 \\
\hline 78 & 11.00345 & 19.59725 & 13.57779 & 11.56316 & 23.49757 & 20.928 & 10.19026 & 20.2031 & 17.60615 \\
\hline
\end{tabular}

Table S9: Verification of observed sample with expected analyte by using thiolfunctionalized quantum dot array in different channels.

\begin{tabular}{|c|c|c|c|c|}
\hline SI No & Expected & Observed (3 Channel) & Observed (6 Channel) & Observed (9 Channel) \\
\hline $\mathbf{1}$ & $2 \mathrm{NP}$ & $2 \mathrm{NP}$ & $2 \mathrm{NP}$ & $2 \mathrm{NP}$ \\
\hline $\mathbf{2}$ & $2 \mathrm{NP}$ & $2 \mathrm{NP}$ & DNA & $2 \mathrm{NP}$ \\
\hline $\mathbf{3}$ & $2 \mathrm{NP}$ & $2 \mathrm{NP}$ & $2 \mathrm{NP}$ & $2 \mathrm{NP}$ \\
\hline $\mathbf{4}$ & $2 \mathrm{NP}$ & $2 \mathrm{NP}$ & $2 \mathrm{NP}$ & $2 \mathrm{NP}$ \\
\hline $\mathbf{5}$ & $2 \mathrm{NP}$ & $2 \mathrm{NP}$ & $2 \mathrm{NP}$ & $2 \mathrm{NP}$ \\
\hline $\mathbf{6}$ & $2 \mathrm{NP}$ & $2 \mathrm{NP}$ & $2 \mathrm{NP}$ & $2 \mathrm{NP}$ \\
\hline $\mathbf{7}$ & MDNB & NOT PREDICTABLE & DNBAL & MDNB \\
\hline $\mathbf{8}$ & MDNB & NOT PREDICTABLE & DNBAL & MDNB \\
\hline $\mathbf{9}$ & MDNB & NOT PREDICTABLE & DNBAL & MDNB \\
\hline $\mathbf{1 0}$ & MDNB & NOT PREDICTABLE & DNBAL & MDNB \\
\hline $\mathbf{1 1}$ & MDNB & NOT PREDICTABLE & DNBAL & MDNB \\
\hline $\mathbf{1 2}$ & MDNB & NOT PREDICTABLE & DNBAL & MDNB \\
\hline $\mathbf{1 3}$ & DNBAL & DNBAL & DNBAL & DNBAL \\
\hline
\end{tabular}




\begin{tabular}{|c|c|c|c|c|}
\hline 14 & DNBAL & DNBAL & DNBAL & DNBAL \\
\hline 15 & DNBAL & DNBAL & DNBAL & DNBAL \\
\hline 16 & DNBAL & DNBAL & DNBAL & DNBAL \\
\hline 17 & DNBAL & DNBAL & DNBAL & DNBAL \\
\hline 18 & DNBAL & DNBAL & DNBAL & DNBAL \\
\hline 19 & DNP & DNP & DNP & DNP \\
\hline 20 & DNP & DNP & DNP & DNP \\
\hline 21 & DNP & DNP & DNP & DNP \\
\hline 22 & DNP & DNP & DNP & DNP \\
\hline 23 & DNP & DNP & DNP & DNP \\
\hline 24 & DNP & DNP & DNP & DNP \\
\hline 25 & DNA & $2 \mathrm{NP}$ & DNA & DNA \\
\hline 26 & DNA & DNA & DNA & DNA \\
\hline 27 & DNA & DNA & DNA & DNA \\
\hline 28 & DNA & NOT PREDICTABLE & DNA & DNA \\
\hline 29 & DNA & $2 A 3 N P$ & DNA & DNA \\
\hline 30 & DNA & NOT PREDICTABLE & DNA & DNA \\
\hline 31 & DNSA & DNSA & DNSA & DNSA \\
\hline 32 & DNSA & DNSA & DNSA & DNSA \\
\hline 33 & DNSA & DNSA & DNSA & DNSA \\
\hline 34 & DNSA & DNSA & DNSA & DNSA \\
\hline 35 & DNSA & DNSA & DNSA & DNSA \\
\hline 36 & DNSA & DNSA & DNSA & DNSA \\
\hline 37 & 4-NA & 4-NA & 4-NA & 4-NA \\
\hline 38 & 4-NA & 2A6NP & 4-NA & 4-NA \\
\hline 39 & 4-NA & NOT PREDICTABLE & 4-NA & 4-NA \\
\hline 40 & 4-NA & 4-NA & 4-NA & 4-NA \\
\hline 41 & 4-NA & 4-NA & 4-NA & 4-NA \\
\hline 42 & 4-NA & 2A6NP & 4-NA & 4-NA \\
\hline 43 & 2A3NP & NOT PREDICTABLE & NOT PREDICTABLE & 2A3NP \\
\hline 44 & $2 A 3 N P$ & NOT PREDICTABLE & NOT PREDICTABLE & 2A3NP \\
\hline 45 & $2 A 3 N P$ & NOT PREDICTABLE & NOT PREDICTABLE & 2A3NP \\
\hline 46 & 2A3NP & NOT PREDICTABLE & NOT PREDICTABLE & 2A3NP \\
\hline 47 & 2A3NP & NOT PREDICTABLE & NOT PREDICTABLE & 2A3NP \\
\hline 48 & 2A3NP & NOT PREDICTABLE & NOT PREDICTABLE & 2A3NP \\
\hline 49 & PA & PA & PA & PA \\
\hline 50 & PA & PA & PA & PA \\
\hline 51 & PA & PA & PA & PA \\
\hline 52 & PA & PA & PA & PA \\
\hline 53 & PA & PA & PA & PA \\
\hline 54 & PA & PA & PA & PA \\
\hline
\end{tabular}




\begin{tabular}{|c|c|c|c|c|}
\hline 55 & CINP & CINP & CINP & CINP \\
\hline 56 & CINP & CINP & MDNB & 2A3NP \\
\hline 57 & CINP & CINP & CINP & CINP \\
\hline 58 & CINP & CINP & MDNB & DNA \\
\hline 59 & CINP & DNBAL & CINP & $2 A 3 N P$ \\
\hline 60 & CINP & $4 \mathrm{NA}$ & DNBAL & DNA \\
\hline 61 & 2A6NP & 2A6NP & 2A6NP & 2A6NP \\
\hline 62 & 2A6NP & 2A6NP & 2A6NP & 2A6NP \\
\hline 63 & $2 A 6 N P$ & NOT PREDICTABLE & 2A6NP & 2A6NP \\
\hline 64 & 2A6NP & 2A6NP & $2 A 6 N P$ & 2A6NP \\
\hline 65 & 2A6NP & 2A6NP & 2A6NP & 2A6NP \\
\hline 66 & 2A6NP & 2A6NP & 2A6NP & 2A6NP \\
\hline 67 & DNBA & DNBA & DNBA & DNBA \\
\hline 68 & DNBA & DNBA & DNBA & DNBA \\
\hline 69 & DNBA & DNBA & DNBA & DNBA \\
\hline 70 & DNBA & DNBA & DNBA & DNBA \\
\hline 71 & DNBA & DNBA & DNBA & DNBA \\
\hline 72 & DNBA & DNBA & DNBA & DNBA \\
\hline 73 & $4 \mathrm{NP}$ & $4 \mathrm{NP}$ & $4 \mathrm{NP}$ & $4 \mathrm{NP}$ \\
\hline 74 & $4 \mathrm{NP}$ & $4 \mathrm{NP}$ & $4 \mathrm{NP}$ & $4 \mathrm{NP}$ \\
\hline 75 & $4 \mathrm{NP}$ & $4 \mathrm{NP}$ & $4 \mathrm{NP}$ & $4 \mathrm{NP}$ \\
\hline 76 & $4 \mathrm{NP}$ & $4 \mathrm{NP}$ & $4 \mathrm{NP}$ & $4 \mathrm{NP}$ \\
\hline 77 & $4 \mathrm{NP}$ & $4 \mathrm{NP}$ & $4 \mathrm{NP}$ & $4 \mathrm{NP}$ \\
\hline 78 & $4 \mathrm{NP}$ & $4 \mathrm{NP}$ & $4 \mathrm{NP}$ & $4 \mathrm{NP}$ \\
\hline
\end{tabular}

Table S10: Comparison of Limits of Detection (LOD) with previous reports

\begin{tabular}{|l|l|l|l|}
\hline Journal & Sensor Elements & Method & LOD $(\mu M)$ \\
\hline
\end{tabular}




\begin{tabular}{|c|c|c|c|}
$\begin{array}{c}\text { Macromolecules 2017, } \\
\text { 50, 4126 }\end{array}$ & Poly(p-phenyleneethynylene)s & $\begin{array}{c}\text { Linear fitting } \\
\text { (Calculated) }\end{array}$ & $4-11$ \\
\hline $\begin{array}{c}\text { ACS Appl. Mater. } \\
\text { Interfaces 2017, 9, } \\
3068\end{array}$ & Truxene based polymer & $\begin{array}{c}\text { Linear fitting } \\
\text { (Calculated) }\end{array}$ & $0.28-2.4$ \\
\hline $\begin{array}{c}\text { APL Materials 2, } \\
124111(\mathbf{2 0 1 4})\end{array}$ & MOF & $\begin{array}{c}\text { Linear fitting } \\
\text { (Calculated) }\end{array}$ & 0.9 \\
\hline $\begin{array}{c}\text { RSC Adv., 2015, } 5, \\
3903\end{array}$ & Phosphorictriamide Scaffold & $\begin{array}{c}\text { Linear fitting } \\
\text { (Calculated) }\end{array}$ & 11 \\
\hline $\begin{array}{c}\text { Anal. Chem. 2018, } 90, \\
12846\end{array}$ & Gold Nanoclusters & $\begin{array}{c}\text { Linear fitting } \\
\text { (Calculated) }\end{array}$ & 0.21 \\
\hline $\begin{array}{c}\text { J. Mater. Chem. C, } \\
\mathbf{2 0 1 6}, 4,6321\end{array}$ & MoS $_{2}$ QDs non functionalized & $\begin{array}{c}\text { Linear fitting } \\
\text { (Calculated) }\end{array}$ & 9 \\
\hline This work & Functionalized QDs & $\begin{array}{c}\text { LDA } \\
\text { (Experimental) }\end{array}$ & 2.5 \\
\hline
\end{tabular}

